Review Article

\title{
Comparative Efficacy and Safety of Metformin, Glyburide, and Insulin in Treating Gestational Diabetes Mellitus: A Meta-Analysis
}

\author{
Lanlan Guo, ${ }^{1}$ Jing Ma $\mathbb{D}^{2},{ }^{2}$ Jia Tang, ${ }^{3}$ Dingyao Hu, ${ }^{4}$ Wei Zhang, ${ }^{5}$ and Xue Zhao ${ }^{6}$ \\ ${ }^{1}$ Department of Radiation Oncology, Sun Yat-sen University Cancer Center, State Key Laboratory of Oncology in South China, \\ Collaborative Innovation Center for Cancer Medicine, Guangzhou 510060, China \\ ${ }^{2}$ Department of Endocrinology and Metabolism, Gansu Provincial Hospital, Lanzhou 730000, China \\ ${ }^{3}$ Department of Infectious Diseases, Huashan Hospital, Fudan University, Shanghai 200041, China \\ ${ }^{4}$ The Second Clinical Medical College, Lanzhou University, Lanzhou 730000, China \\ ${ }^{5}$ Department of Critical Care Medicine, Affiliated Hospital of Zunyi Medical College, Zunyi 563000, China \\ ${ }^{6}$ Department of Nephrology, Shandong Provincial Hospital Affiliated to Shandong University, Jinan 250021, China
}

Correspondence should be addressed to Jing Ma; treasuremj@163.com

Received 8 May 2019; Revised 3 August 2019; Accepted 11 August 2019; Published 4 November 2019

Academic Editor: Andrea Scaramuzza

Copyright (c) 2019 Lanlan Guo et al. This is an open access article distributed under the Creative Commons Attribution License, which permits unrestricted use, distribution, and reproduction in any medium, provided the original work is properly cited.

To compare the efficacy and safety of metformin, glyburide, and insulin in treating gestational diabetes mellitus (GDM), a meta-analysis of randomized controlled trials (RCTs) was conducted. PubMed, Embase, CINAHL, Web of Science, and Cochrane Library to November 13, 2018, were searched for RCT adjusted estimates of the efficacy and safety of metformin, glyburide, and insulin treatments in GDM patients. There were 41 studies involving 7703 GDM patients which were included in this meta-analysis; 12 primary outcomes and 24 secondary outcomes were detected and analyzed. Compared with metformin, insulin had a significant increase in the risk of preeclampsia (RR, 0.57; 95\% CI, 0.45 to $0.72 ; P<0.001$ ), NICU admission (RR, $0.75 ; 95 \% \mathrm{CI}, 0.64$ to $0.87 ; P<0.001$ ), neonatal hypoglycemia (RR, $0.57 ; 95 \% \mathrm{CI}, 0.49$ to $0.66 ; P<0.001$ ), and macrosomia (RR, 0.68 ; $95 \%$ CI, 0.55 to $0.86 ; P<0.05$ ). To the outcomes of birth weight and gestational age at delivery, insulin had a significant increase when compared with metformin (MD, 114.48; 95\% CI, 37.32 to 191.64; $P<0.01$; MD, 0.23 ; $95 \%$ CI, 0.12 to 0.34 ; $P<0.001$; respectively). Of the two groups between glyburide and metformin, metformin had lower gestational weight gain compared with glyburide (MD, 1.67; 95\% CI, 0.26 to 3.07; $P<0.05$ ). Glyburide had a higher risk of neonatal hypoglycemia compared with insulin (RR, 1.76; $95 \% \mathrm{CI}, 1.32$ to 2.36 ; $P<0.001)$. This meta-analysis found that metformin could be a safe and effective treatment for GDM. However, clinicians should pay attention on the long-term offspring outcomes of the relative data with GDM patients treated with metformin. Compared with insulin, glyburide had a higher increase of neonatal hypoglycemia. The use of glyburide in pregnancy for GDM women appears to be unclear.

\section{Introduction}

Gestational diabetes mellitus (GDM) is the most frequent medical complication of pregnancy and becoming a major global public health issue with the increasing prevalence in recent years due to the epidemic of obesity and type 2 diabetes. GDM affects about 7\% of pregnancies in North America and has a global prevalence range from $5.8 \%$ to $12.9 \%$ and is associated with several maternal and neonatal adverse outcomes [1]. The presence of GDM always accompanies an increased maternal risk for preeclampsia and cesarean section and with an increased risk for developing type 2 diabetes (T2D) after pregnancy [2]. Moreover, GDM increases the risk of macrosomia, large for gestational age, shoulder dystocia, birth injury, neonatal hypoglycemia, preterm birth, hyperbilirubinemia, and others $[3,4]$. Treatment of GDM can prevent short-term maternal and neonatal complications. The initial management for GDM includes nutritional modification and physical activity [5]. Almost $30 \%$ of women with GDM cannot be managed with diet and lifestyle 
modification alone and require pharmacological therapy to reduce the associated maternal and neonatal short- and long-term effects of GDM $[6,7]$.

Insulin historically has been considered the standard therapy for GDM management in cases refractory to nutrition therapy and exercise $[7,8]$, and this has continued to be reinforced by the ADA [8]. Insulin, which does not cross the placenta, lowers blood glucose by stimulating peripheral glucose uptake and inhibiting glucose production release by the liver [9]. However, it requires multiple daily injections and subsequently the need to train the patients in the technical aspect of treatment, resulting to more weight gain and higher medical cost [10-15]. In addition, hypoglycemia occurs in approximately $70 \%$ of women who use insulin some time during their pregnancy [16].

Oral antihyperglycemic drugs (OADs) (such as metformin and glyburide) can cross the placenta to the fetus. In addition, all oral agents lack long-term safety data. Therefore, they have not been approved by the U.S. Food and Drug Administration [17] and insulin continues to be the ADA recommended first-line therapy [8].

Metformin, as the first-line medication for T2DM, can promote glucose level control and lose weight and improve peripheral insulin resistance. Metformin is also known to increase the secretion of glucagon-like peptide 1 (GLP-1) from intestinal cells [18]. It is increasingly recognized as an alternative to insulin therapy for $\operatorname{GDM}[19,20]$. However, metformin has been found to have a maternal to fetal transfer and the long-term influence is uncertain. The largest study of metformin was in the MiG (metformin use in GDM) trial; Rowan et al. found that the primary composite outcome of neonatal morbidity was similar in the metformin arm compared to the insulin arm. Moreover, severe neonatal hypoglycemia was lower compared to women on insulin alone $[21,22]$.

Glyburide can stimulate the release of insulin from the pancreas. According to the recent study, Song et al. [23] reported that no significant differences in maternal shortterm outcomes were observed between glyburide and insulin groups. Glyburide is a second-generation SU that can be considered safe and effective for the treatment of GDM. However, there are some concerns regarding a higher risk of macrosomia, large-for-gestational age infants, and neonatal hypoglycemia compared to insulin. Data regarding its use in GDM are conflicting in several studies [16, 23-29].

Because there is the paucity of adequate safety data, the use of these two drugs in GDM is restricted to the USA, although they are increasingly used now in Europe and South Africa [30]. In the past, oral hypoglycemic agents including metformin and glyburide have been used as alternative pharmacological treatment to insulin therapy [21, 31]. Nonetheless, the most recent 2019 American Diabetes Association guidelines do not recommend metformin and glyburide as first-line treatment for GDM, because they are known to cross the placenta and data on safety for offspring is lacking $[32,33]$. In addition, the 2014 German Diabetes Association and German Association of Gynecology and Obstetrics guidelines do not recommend the use of oral hypoglycemic agents in GDM [34].
In recent years, several meta-analyses sought to assess the efficacy and safety of the treatment in GDM patients. In 2014, Jiang et al.'s study [14] including 18 RCTs revealed that both metformin and glyburide are suitable for use in the management of GDM, but glyburide was associated with more adverse pregnancy outcomes, including neonatal hypoglycemia, high maternal weight gain, high neonatal birth weight, and macrosomia. In 2017, Liang et al.'s study [28] including 31 RCTs revealed that metformin had more favorable pregnancy outcomes and the fastest rate of glucose control, especially in obese GDM patients, but with the lowest rate of average glucose control; glyburide have the highest rate of average glucose control, particularly in nonobese GDM patients, but with more adverse outcomes. The efficacy and safety of insulin, metformin, and glyburide in the treatment of GDM remain to be debated. Therefore, we performed a meta-analysis with the updated data, which might provide more evidence with respect to the efficacy and safety of metformin, glyburide, and insulin.

\section{Materials and Methods}

2.1. Ethic Statement. The protocol of this systematic review was registered in PROSPERO database on 8 March 2019 (CRD42019122611). This study was conducted according to the Cochrane Handbook for Systematic Reviews of Interventions [35], and the results were presented according to the PRISMA statement [36].

2.2. Search Strategy. A comprehensive electronic search strategy was conducted in PubMed, Embase, Web of Science, CINAHL, and Cochrane Library up to November 13, 2018. Authors of potentially eligible studies were contacted when necessary to request further information regarding study design or primary outcomes. The search strategies are included in Table 1.

2.3. Selection Criteria. Studies were included if they met the following criteria: subjects were women with gestational diabetes requiring drug treatment; the study was a randomized controlled trial that compares efficacy and safety parameters of metformin, glyburide, or insulin; the study provided information on one or more maternal or fetal outcome; they were published as a full paper. The exclusion criteria were as follows: reviews, letters, conferences abstract, case reports or series, comments, and animal experiment. Studies involving pregnant women with preexisting diabetes and studies with duplicated data were excluded.

2.4. Data Extraction and Quality Assessment. Two review authors independently assessed the quality of each included study by using the tool in the Cochrane Handbook for Systematic Reviews of Intervention. Two reviewers (Guo and $\mathrm{Ma}$ ) independently performed the literature search, study selection, and data extraction. Differences in opinions were resolved by consensus with a third reviewer (Tang). When necessary, we contacted authors of original studies for additional data. 
TABle 1: Search strategy.

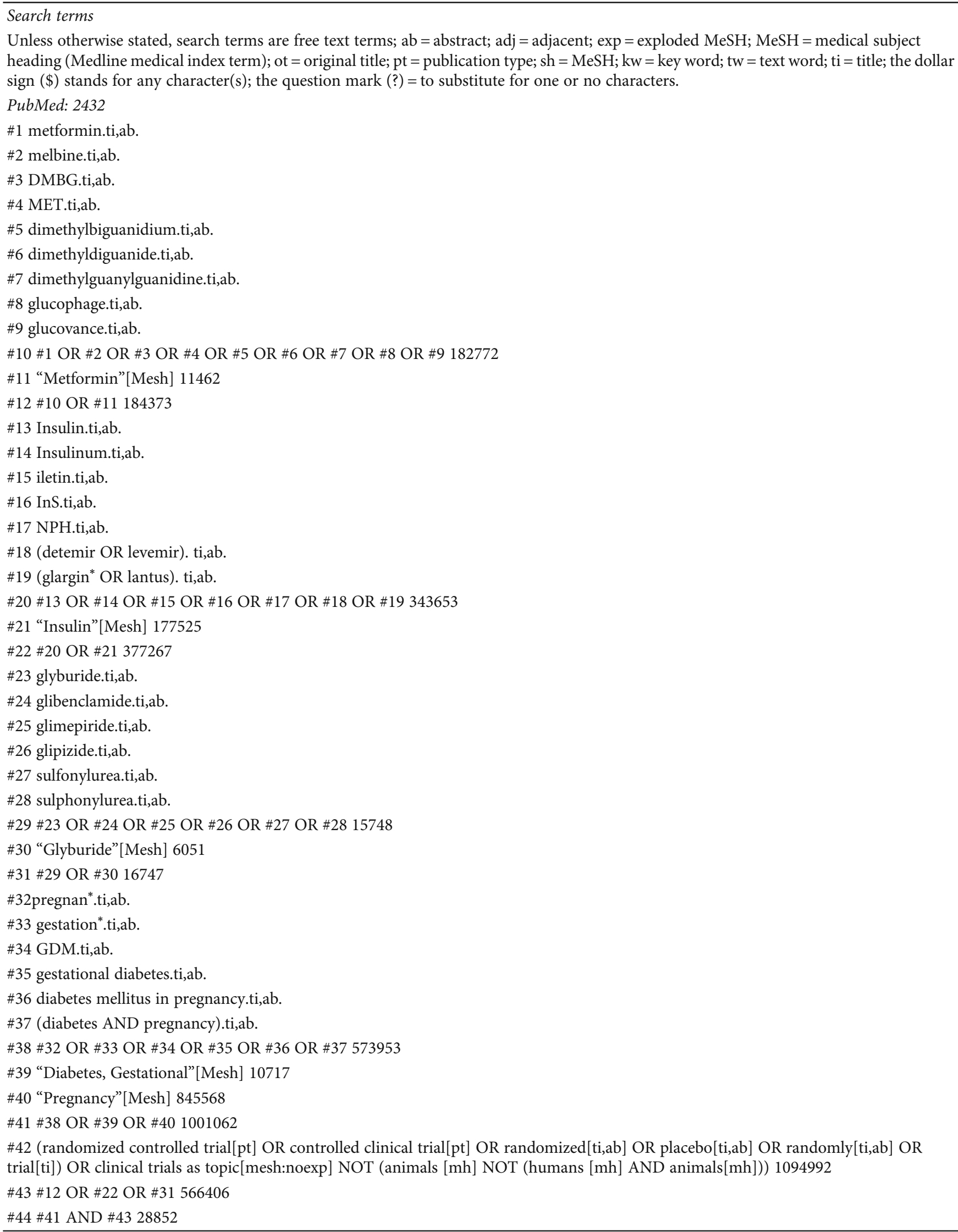


TABLE 1: Continued.

\#45 \#44 AND \#42 2432

Embase: 3978

\#1 'metformin'/exp 55,211

\#2 ‘metformin':ab,ti OR 'melbine’:ab,ti OR ‘dmbg’:ab,ti OR ‘met’:ab,ti OR ‘dimethylbiguanidium':ab,ti OR ‘dimethyldiguanide’:ab,ti OR 'dimethylguanylguanidine':ab,ti OR 'glucophage':ab,ti OR 'glucovance':ab,ti 285,081

\#3 \#1 OR \#2 312,295

\#4 'insulin'/exp 335,113

\#5 'insulinum':ab,ti OR 'insulin':ab,ti OR 'nph':ab,ti OR 'detemir':ab,ti OR 'levemir':ab,ti OR 'glargin*':ab,ti OR 'lantus':ab,ti 460,703

\#6 \#4 OR \#5 543,932

$\# 7$ 'glibenclamide'/exp 23,962

\#8 'glyburide’:ab,ti OR 'glibenclamide’:ab,ti OR 'glimepiride’:ab,ti OR 'glipizide’:ab,ti OR 'sulfonylurea':ab,ti OR ‘sulphonylurea':ab,ti 23,059

\#9 \#7 OR \#8 34,460

\#10 'pregnancy'/exp 745,337

\#11 'pregnancy diabetes mellitus'/exp 32,173

\#12 'pregnan*':ab,ti OR 'gestation*':ab,ti OR 'gdm':ab,ti OR 'gestational diabetes':ab,ti OR 'diabetes mellitus in pregnancy':ab,ti OR ('diabetes':ab,ti AND 'pregnancy':ab,ti) 770,703

\#13 \#10 OR \#11 OR \#12 1,057,118

\#14 \#3 OR \#6 OR \#9 845,415

\#15 \#13 AND \#14 40,184

\#16 \#15 AND ([cochrane review]/lim OR [systematic review]/lim OR [meta analysis]/lim OR [controlled clinical trial]/lim OR [randomized controlled trial]/lim) 3978

Web of Science: 4651

\# 1 Topic: (Metformin) OR Topic: (melbine) OR Topic: (DMBG) OR Topic: (MET) OR Topic: (dimethylbiguanidium) OR Topic: (dimethyldiguanide) OR Topic: (glucophage) OR Topic: (glucovance) 576,285

\# 2 Topic: (Insulin) OR Topic: (iletin) OR Topic: (InS) OR Topic: (NPH) OR Topic: (detemir or levemir) OR Topic: (glargin* or lantus) 437,814

\# 3 Topic: (glyburide) OR Topic: (glibenclamide) OR Topic: (glimepiride) OR Topic: (glipizide) OR Topic: (sulfonylurea) OR Topic: (sulphonylurea) 19,751

\# 4 Topic: (Pregnancy) OR Topic: (Diabetes, Gestational) OR Topic: (pregnan*) OR Topic: (gestation*) OR Topic: (GDM) OR Topic: (gestational diabetes) OR Topic: (diabetes mellitus in pregnancy) OR Topic: (diabetes AND pregnancy) 488,448

\# 5 Topic: (randomized controlled trial) OR Topic: (controlled clinical trial) OR Topic: (randomized) OR Topic: (placebo) OR Topic: (randomly) OR Topic: (trial) OR Topic: (clinical trials as topic) 1,921,062

\# 6 \#3 OR \#2 OR \#1 1,027,299

\# 7 \#4 AND \#6 28,082

\# 8 \#5 AND \#7 4,651

CINAHL: 878

\#1 (MH “Metformin”) 4,429

\#2 (AB Metformin OR AB melbine OR AB DMBG OR AB MET OR AB dimethylbiguanidium OR AB dimethyldiguanide OR AB dimethylguanylguanidine $\mathrm{OR} A B$ glucophage $\mathrm{OR} \mathrm{AB}$ glucovance) 55,171

\#3 (MH “Insulin+") 26,217

\#4 (MH “Insulin, Short-Acting”) 51

\#5 (MH “Insulin, Intermediate-Acting”)20

\#6 (MH “Insulin, Long-Acting”)328

\#7 (MH “Insulin, Rapid-Acting+”)294

\#8 (MH "Protamines") 181

\#9 AB Insulin OR AB Insulinum OR AB iletin OR AB InS OR AB NPH OR AB neutral protamine hegedom OR AB (detemir or levemir) OR $\mathrm{AB}$ (glargin* or lantus) 37,056

\#10 (MH “Glyburide") 607

\#11 (MH “Glimepiride”) 82

\#12 (MH “Glipizide") 138

\#13 (MH “Sulfonylurea Compounds+") 2,146 
TABLe 1: Continued.

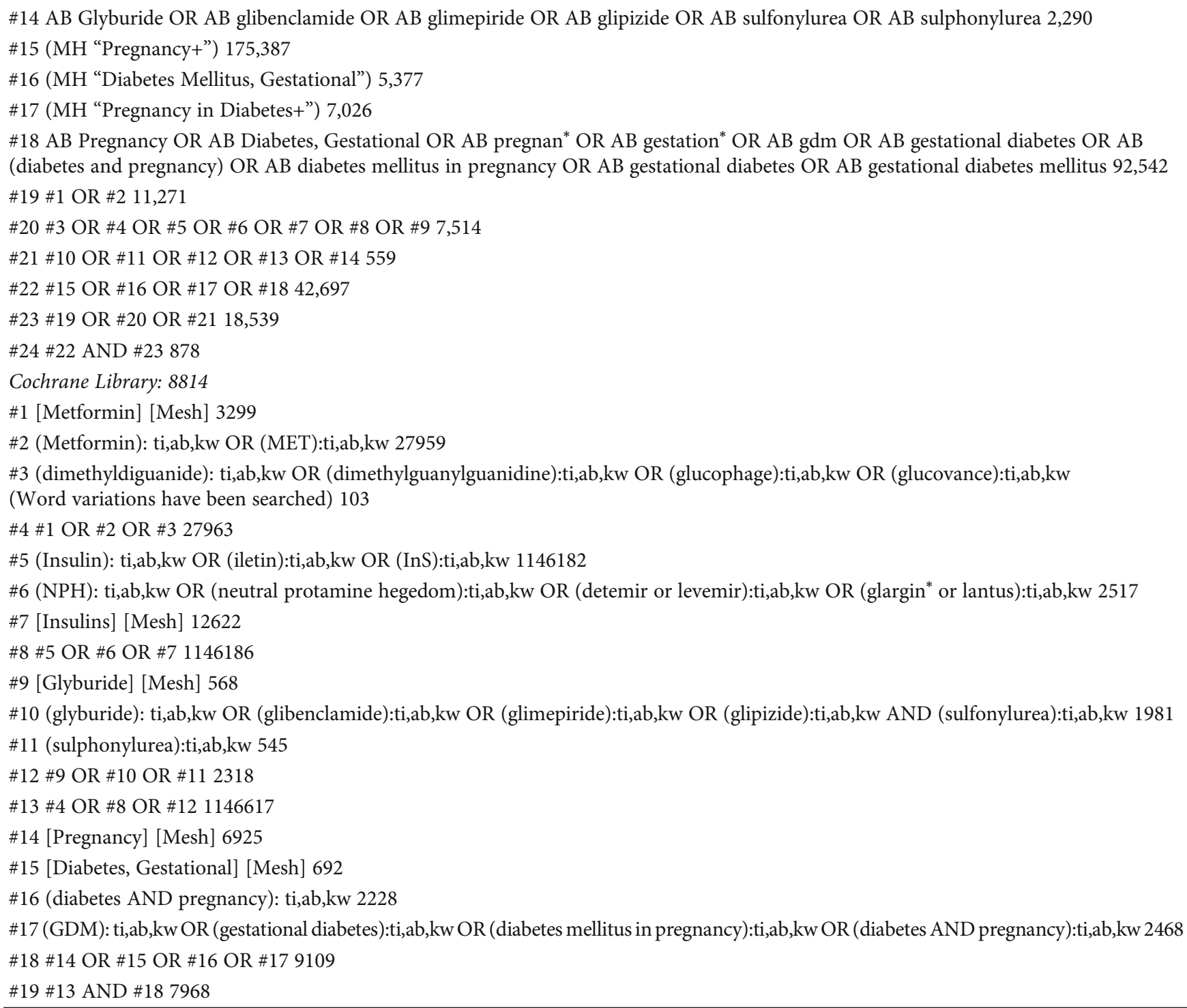

2.5. Outcomes of Interest. Outcomes of interest were divided into 2 categories: neonatal outcomes and maternal outcomes. There are 18 neonatal outcomes, including neonatal intensive care unit (NICU) admission, neonatal hypoglycemia $(<2.2 \mathrm{mmol} / \mathrm{L})$, macrosomia $(>4 \mathrm{~kg})$, sepsis, and respiratory distress syndrome (RDS). There are 17 maternal outcomes, including preeclampsia (blood pressure $>140 / 90 \mathrm{mmHg}$ with proteinuria $>0.3 \mathrm{~g} / 24 \mathrm{~h}$ ), gestational hypertension, mode of delivery, maternal hypoglycemia $(<3.3 \mathrm{mmol} / \mathrm{L})$, pregnancyinduced hypertension (PIH), gestational weight gain, and $\mathrm{HbAlc} \%$.

2.6. Statistical Analysis. All analyses were performed using Review Manager 5.3 (Nordic Cochrane Centre). A fixedeffects model was used to pool the data if no significant heterogeneity was reported, and a random-effects model was used in the case of significant heterogeneity being used for an outcome, to calculate the risk ratio (RR) or mean difference (MD) and to assess the neonatal and maternal outcomes of different treatments in GDM patients. For continuous outcomes, we calculated mean differences (MD) and 95\% confidence intervals (CI). For dichotomous outcomes, we calculated risk ratio (RR) and 95\% CI. The heterogeneity was evaluated statistically by the chi-squared test $\left(P<0.1, I^{2}<50 \%\right)$ and graphically using a forest or funnel plot analysis. If $I^{2}>50 \%$, a random-effects model was used for the meta-analysis; if $I^{2}<50 \%$, a fixed-effects model was used for the meta-analysis. $P<0.05$ is considered to be statistically significant.

\section{Results}

3.1. Search Results. The search retrieved 19907 abstracts. There were 15225 studies after duplicates were removed. Eventually, 41 studies fulfilled our inclusion criteria-23 comparing metformin with insulin (4674 subjects), 13 comparing 


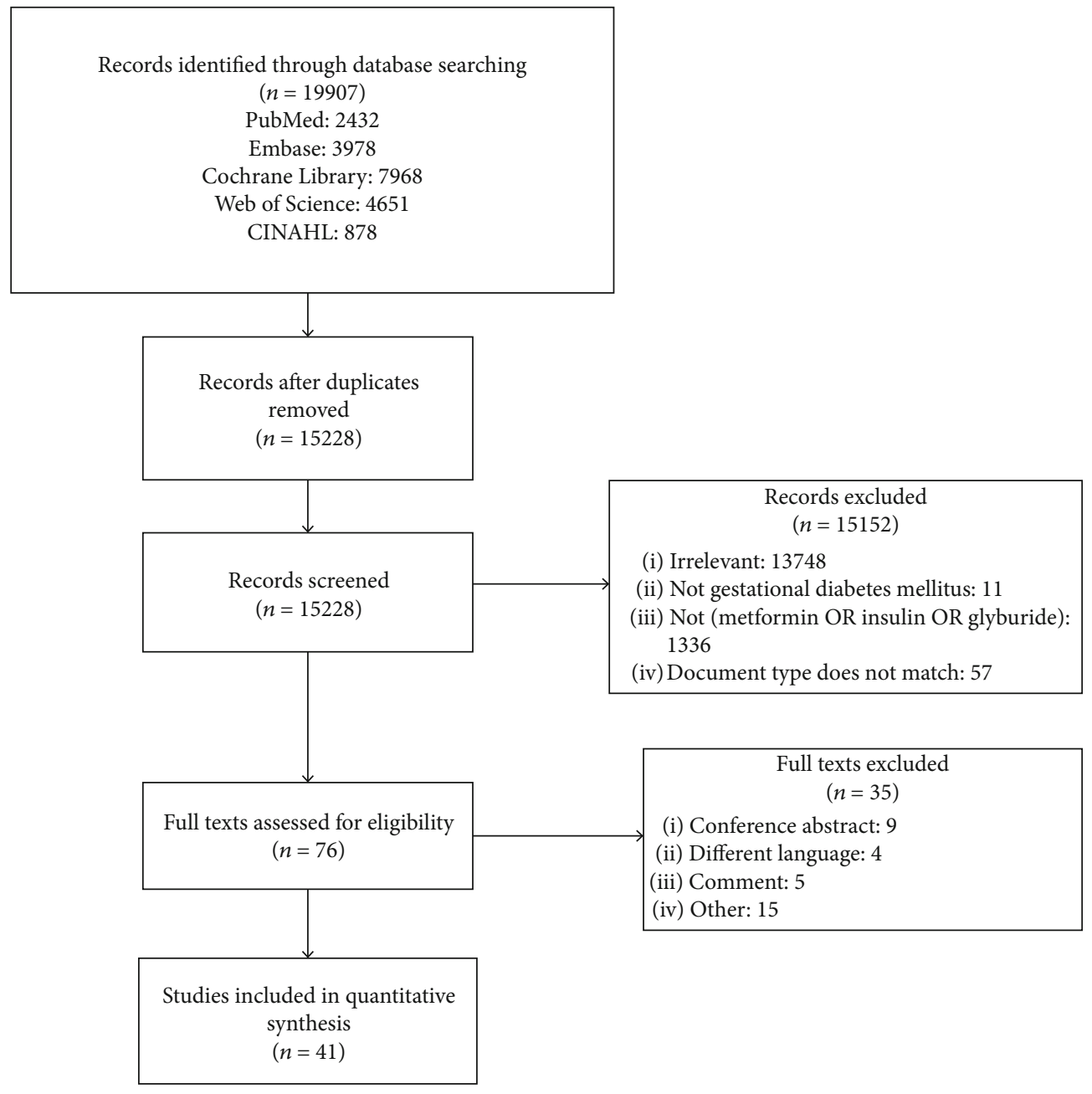

FIgURE 1: The search flow diagram.

glibenclamide with insulin (2561subjects), and 5 comparing metformin with glibenclamide (684 subjects) $[21,24,25,29$, $31,37-72]$. Figure 1 shows the search flow diagram. The characteristics of the included studies are described in Table 2.

3.2. Assessment of Risk of Bias. Due to the high risk of bias, the results from the aforementioned studies were analyzed separately as required to determine whether the conclusions were affected by the inclusion of these "highrisk" studies. Two review authors independently assessed the risk of bias for each included study by using the Cochrane Collaboration's risk-of-bias tool in the Cochrane Handbook for Systematic Reviews of Interventions [73]. The quality assessments of the included studies are presented in Figures 2 and 3.

3.3. Effects of Intervention. In the forest and funnel plots, studies were categorized into the following groups: "metformin vs. insulin," "glyburide vs. insulin," and "glyburide vs. metformin." The complete set of forest plots and funnel plots are available in the appendix.

3.3.1. Preterm Birth. Preterm birth was included as an outcome by 11 studies which involved 2943 GDM patients.
There was significant heterogeneity between these studies $\left(P<0.001, I^{2}=71 \%\right)$. The pooled result showed no significant statistical difference between the metformin and insulin groups in terms of preterm birth (RR, 0.90; 95\% CI, 0.51 to 1.58; $P=0.71)$. Preterm birth was reported as an outcome between glyburide and insulin by 3 studies which included 941 GDM patients. There was no significant heterogeneity between these studies $\left(P=0.81, I^{2}=0 \%\right)$. There was no significant statistical difference between the glyburide and insulin groups in terms of preterm birth (RR, 1.58; 95\% CI, 0.90 to $2.76 ; P=0.11$ ).

\subsubsection{Hypertensive Disorders}

(1) Gestational Hypertension. Gestational hypertension was included as an outcome by 5 studies which involved 1388 GDM patients. There was no significant heterogeneity between 5 studies $\left(P=0.67, I^{2}=0 \%\right)$. In the pairwise metaanalysis, we observed that metformin had lower incidence of preeclampsia compared with insulin (RR, 0.56; 95\% CI, 0.36 to $0.87 ; P<0.01)$.

(2) Pregnancy-Induced Hypertension (PIH). Pregnancyinduced hypertension was included as an outcome between 


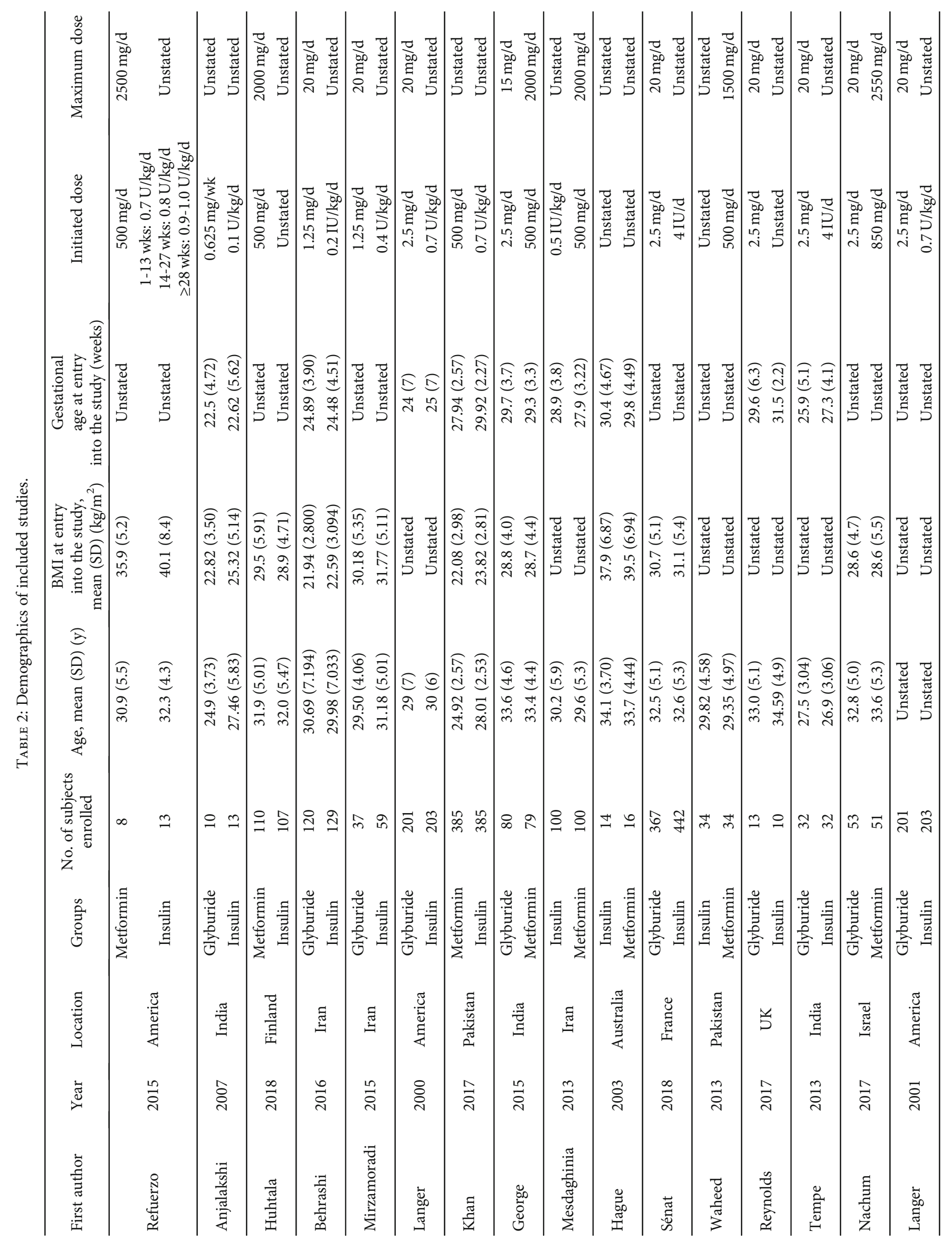




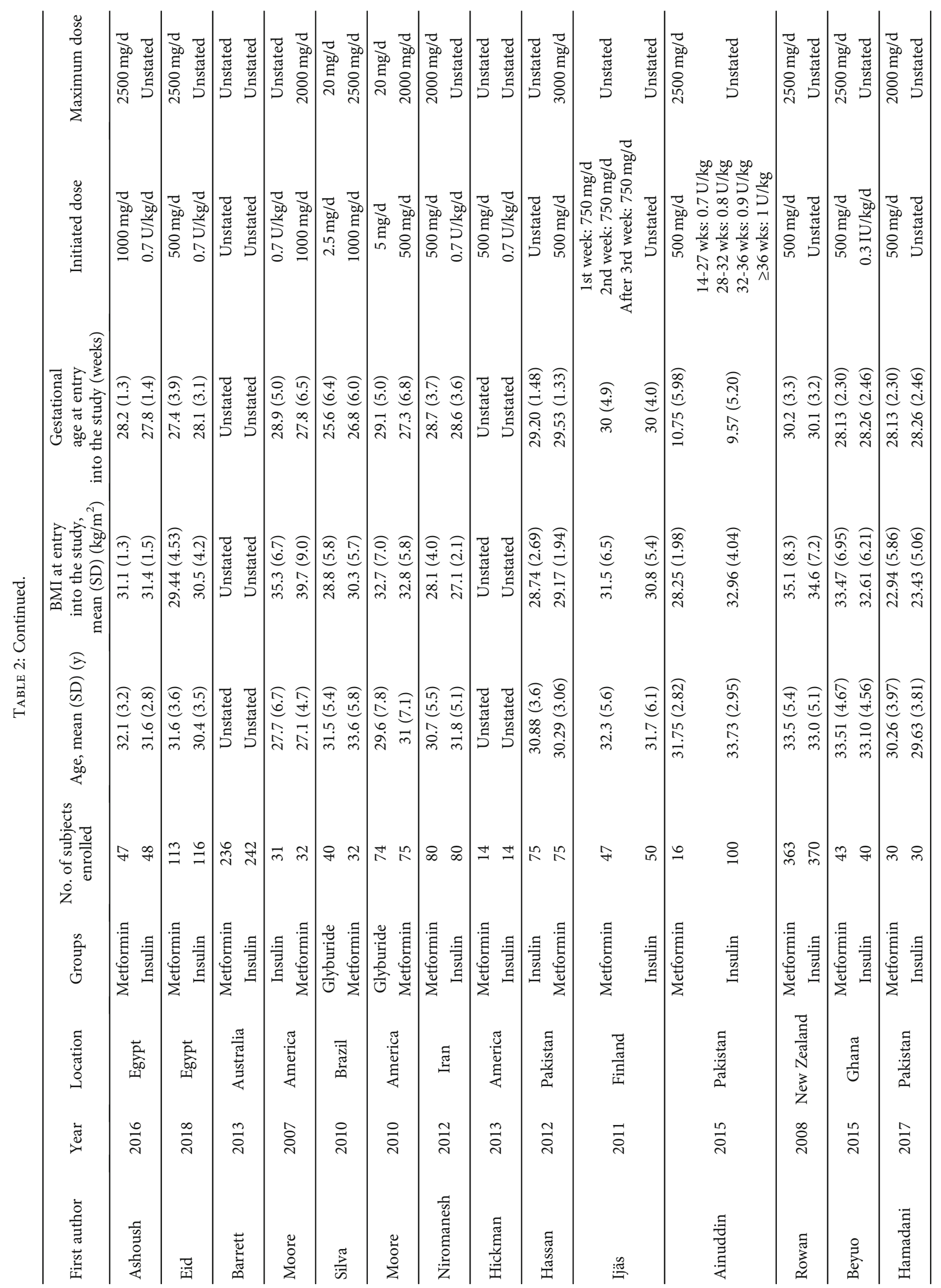




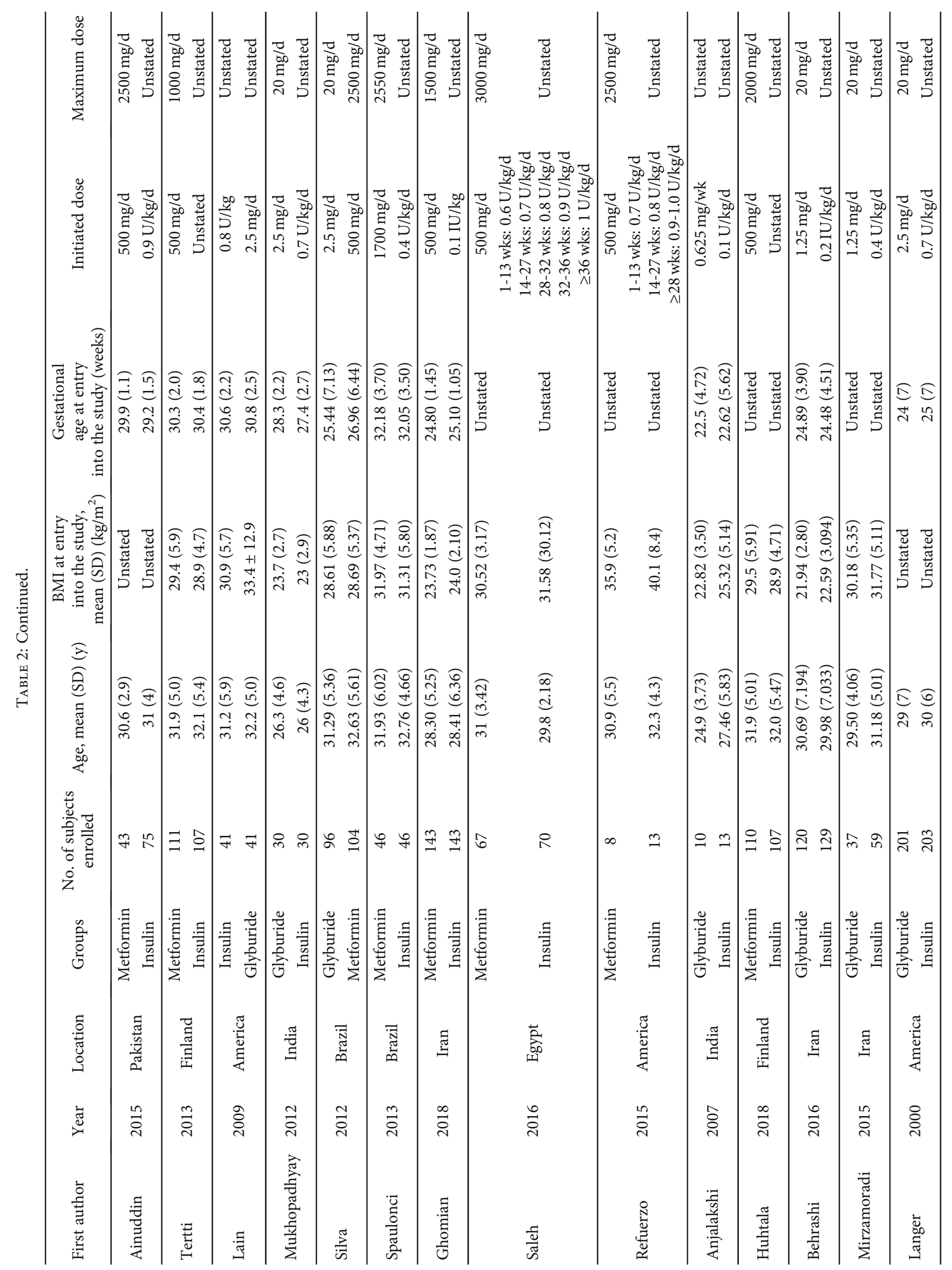




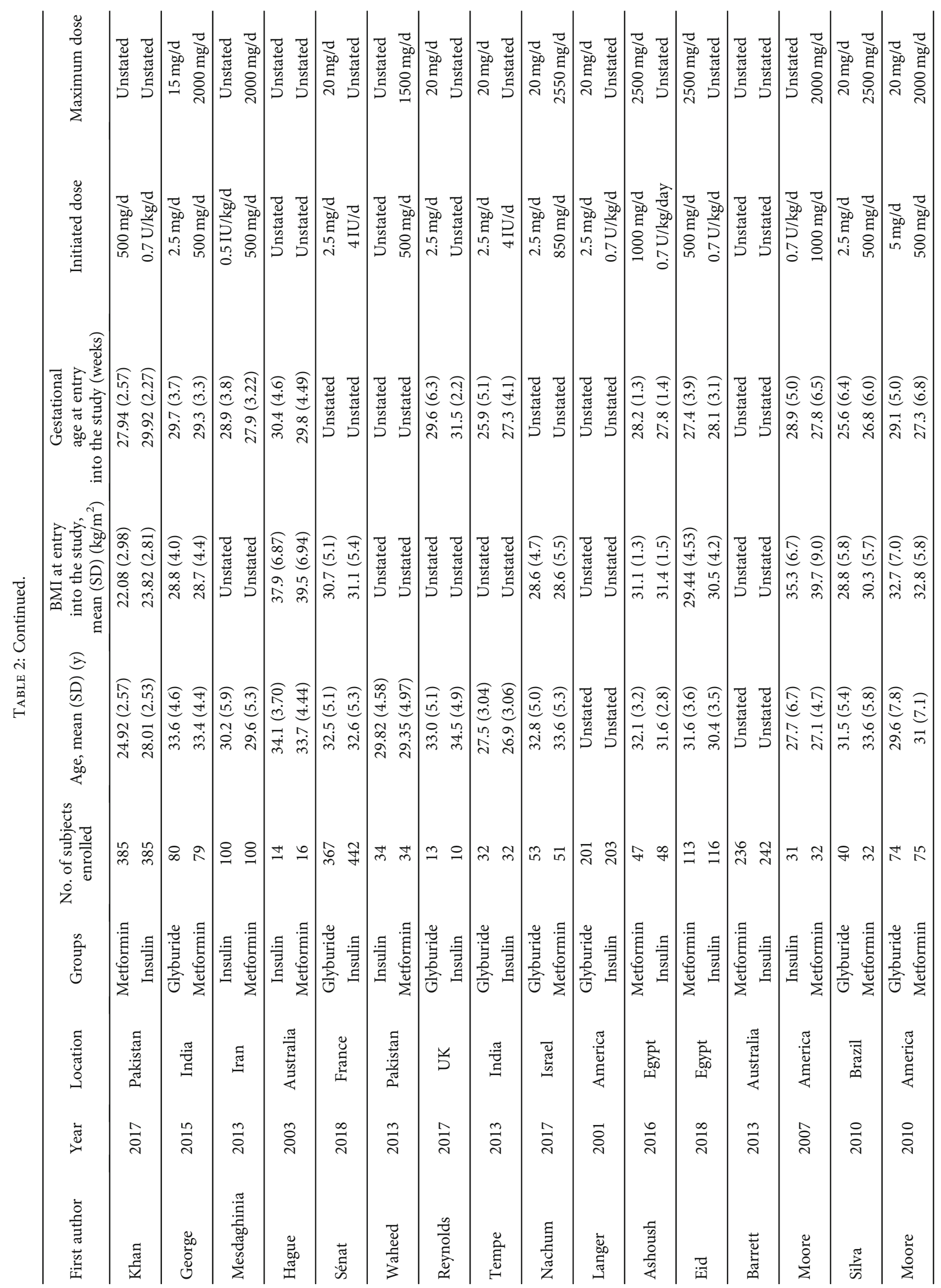




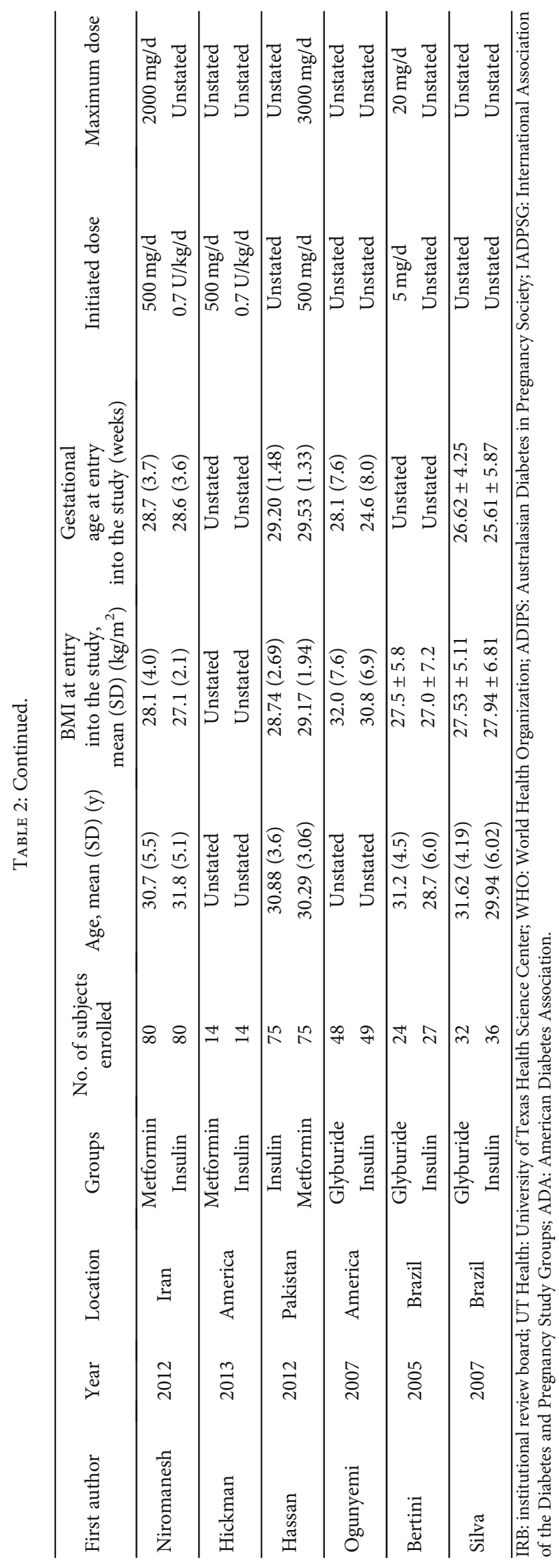




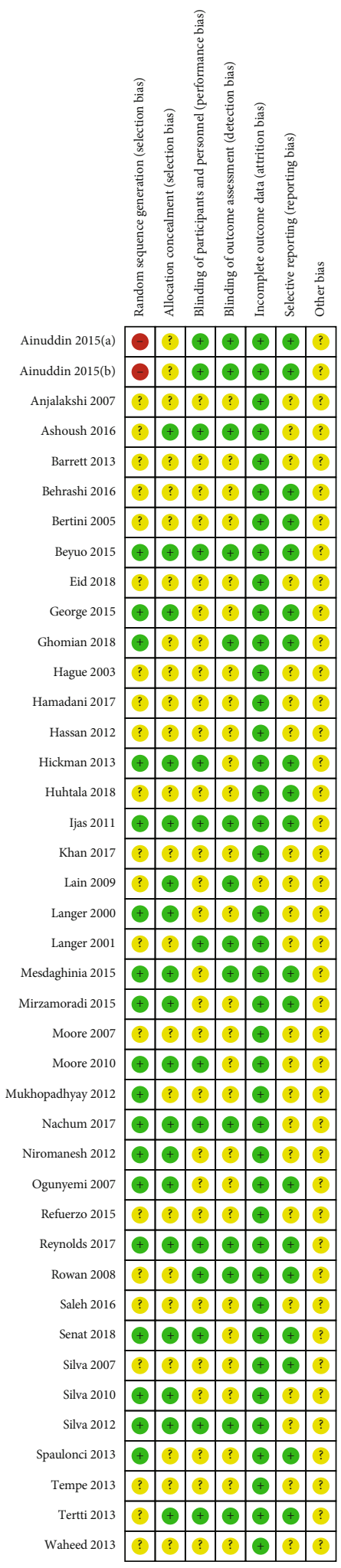

FIGURE 2: Risk of bias summary.

metformin and insulin by 3 studies which involved 606 GDM patients. There was no significant heterogeneity between these studies $\left(P=0.52, I^{2}=0 \%\right)$. Data showed no significant statistical difference between the metformin and insulin groups in terms of pregnancy-induced hypertension (RR, 0.56 ; $95 \%$ CI, 0.30 to 1.06 ; $P=0.08$ ). 


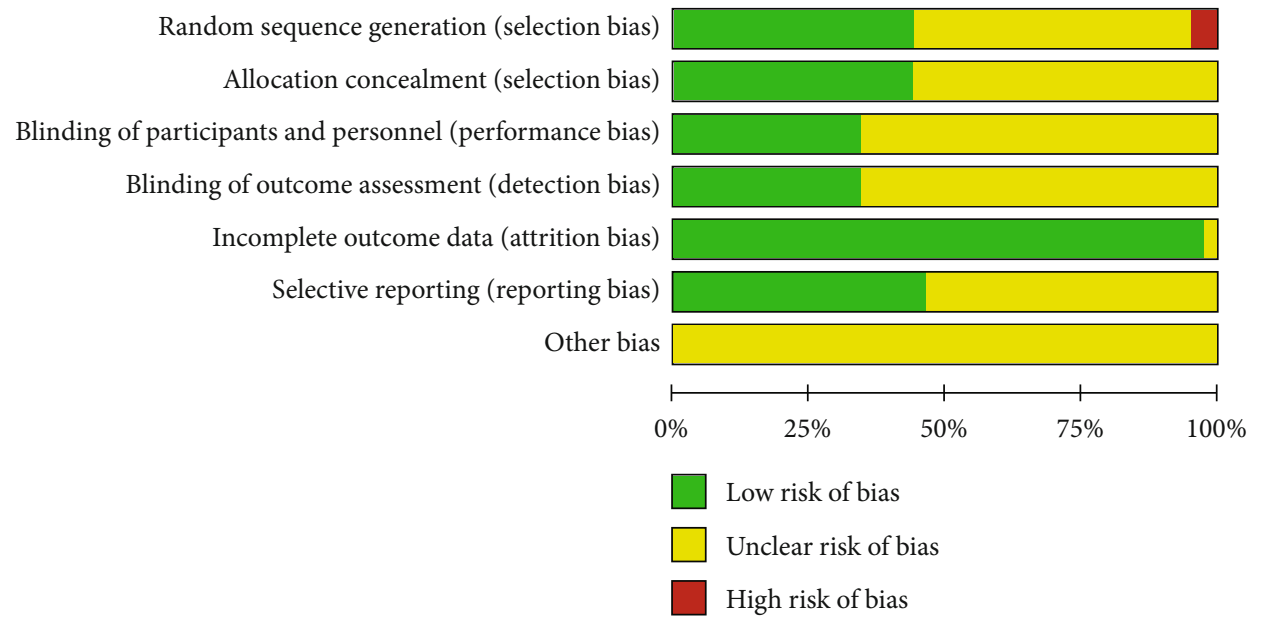

Figure 3: Risk of bias graph.

(3) Preeclampsia. Preeclampsia was included as an outcome between metformin and insulin by 14 studies which involved 3402 GDM patients. There was no significant heterogeneity between these studies $\left(P=0.05, I^{2}=43 \%\right)$. In the pairwise meta-analysis, we observed that metformin had lower incidence of preeclampsia than insulin (RR, 0.57; 95\% CI, 0.45 to $0.72 ; P<0.001$ ). Three studies involving $564 \mathrm{GDM}$ patients focused on the incidence of preeclampsia between glyburide and insulin. There was no significant heterogeneity between these studies $\left(P=0.58, I^{2}=0 \%\right)$. The pooled result showed no significant statistical difference between the glyburide and insulin groups in terms of preeclampsia (RR, 0.98 ; $95 \%$ CI, 0.56 to $1.74 ; P=0.95)$.

\subsubsection{Mode of Delivery}

(1) Induction of Labor. Induction of labor was included as an outcome by 8 studies which involved 1066 GDM patients. There was no significant heterogeneity between these studies $\left(P=0.10, I^{2}=42 \%\right)$. In the pairwise meta-analysis, we observed that metformin was associated with a significantly reduced incidence of induction of labor compared with insulin (RR, 0.85 ; $95 \%$ CI, 0.74 to $0.99 ; P<0.05$ ).

(2) Cesarean Section. Cesarean section was included as an outcome between metformin and insulin by 15 studies which involved 2611 GDM patients. There was no significant heterogeneity between these studies $\left(P=0.12, I^{2}=31 \%\right)$. There was no significant statistical difference between the metformin and insulin groups in terms of cesarean section (RR, 1.00 ; $95 \% \mathrm{CI}, 0.90$ to $1.10 ; P=0.96$ ). Five studies involving 1429 GDM patients reported the cesarean section between glyburide and insulin. There was no significant heterogeneity between these studies $\left(P=0.70, I^{2}=0 \%\right)$. The pooled result showed no significant statistical difference between the glyburide and insulin groups in terms of cesarean section (MD, $0.89 ; 95 \%$ CI, 0.71 to $1.13 ; P=0.35$ ). Four studies involving 525 GDM patients focused on the incidence of cesarean section between glyburide and metformin.
There was significant heterogeneity between these studies $\left(P=0.10, I^{2}=52 \%\right)$. The pooled result showed no significant statistical difference between the glyburide and metformin groups in terms of cesarean delivery (RR, 0.95; 95\% CI, 0.71 to $1.27 ; P=0.73$ ).

(i) Elective Cesarean Section. Elective cesarean section was included as an outcome between metformin and insulin by 3 studies which involved 526 GDM patients. There was no significant heterogeneity between these studies $(P=0.16$, $\left.I^{2}=46 \%\right)$. In the pairwise meta-analysis, we observed that metformin had lower incidence of elective cesarean section compared with insulin (RR, 0.73; 95\% CI, 0.54 to 1.00 ; $P=0.05)$.

(ii) Emergency Cesarean Section. Emergency cesarean section was included as an outcome between metformin and insulin by 3 studies which involved 526 GDM patients. There was no significant heterogeneity between these studies $(P=0.32$, $\left.I^{2}=13 \%\right)$. However, there was no significant statistical difference between the metformin and insulin groups in terms of emergency cesarean section (RR, 1.10; 95\% CI, 0.82 to $1.49 ; P=0.52)$.

(3) Vaginal Delivery. Vaginal delivery was included as an outcome between metformin and insulin by 8 studies which involved 1206 GDM patients. There was no significant heterogeneity between these studies $\left(P=0.10, I^{2}=42 \%\right)$. However, there was no significant statistical difference between the metformin and insulin groups in terms of vaginal delivery (RR, $1.12 ; 95 \% \mathrm{CI}, 0.99$ to $1.25 ; P=0.06$ ).

(i) Assisted Vaginal Delivery. Assisted vaginal delivery was included as an outcome between metformin and insulin by 4 studies which involved 667 GDM patients. There was no significant heterogeneity between these studies $(P=0.70$, $\left.I^{2}=0 \%\right)$. However, there was no significant statistical difference between the metformin and insulin groups in terms of 
assisted vaginal delivery $(\mathrm{RR}, 1.06 ; 95 \% \mathrm{CI}, 0.63$ to 1.80 ; $P=0.82)$.

(ii) Spontaneous Vaginal Delivery. Spontaneous vaginal delivery was included as an outcome between metformin and insulin by 4 studies which involved 680 GDM patients. There was significant heterogeneity between these studies $\left(P=0.04, I^{2}=65 \%\right)$. The pooled result showed no significant statistical difference between the metformin and insulin groups in terms of spontaneous vaginal delivery $(R R, 0.97$; $95 \% \mathrm{CI}, 0.54$ to $1.74 ; P=0.96)$.

3.3.4. Maternal Hypoglycemia. Maternal hypoglycemia was included as an outcome between metformin and insulin by 3 studies which involved 352 GDM patients. There was no significant heterogeneity between these studies $(P=0.49$, $\left.I^{2}=0 \%\right)$. In the pairwise meta-analysis, we observed that metformin had lower incidence of maternal hypoglycemia compared with insulin (RR, $0.28 ; 95 \% \mathrm{CI}, 0.10$ to $0.75 ; P=0.05)$.

3.3.5. Gestational Age at Delivery. Cesarean delivery was included as an outcome between insulin and metformin by 12 studies which involved 2295 GDM patients. There was no significant heterogeneity between these studies $(P=0.55$, $\left.I^{2}=0 \%\right)$. In the pairwise meta-analysis, we observed that metformin had lower gestational age at delivery compared with insulin (MD, 0.23; 95\% CI, 0.12 to $0.34 ; P<0.001$ ). Seven studies involving 1007 GDM patients reported the gestational age at delivery between glyburide and insulin. There was significant heterogeneity between these studies $\left(P<0.001, I^{2}=79 \%\right)$. The pooled result showed no significant statistical difference between the glyburide and insulin groups in terms of gestational age at delivery (MD, 0.14; $95 \% \mathrm{CI},-0.32$ to $0.61 ; P=0.55)$. Four studies involving 535 GDM patients reported the gestational age at delivery between glyburide and metformin. There was no significant heterogeneity between these studies $\left(P=0.17, I^{2}=40 \%\right)$. However, the pooled result showed no significant statistical difference between the glyburide and metformin groups in terms of gestational age at delivery ( $\mathrm{MD}, 0.10 ; 95 \%$ CI, -0.13 to $0.33 ; P=0.39)$.

3.3.6. Gestational Weight Gain. Gestational weight gain was included as an outcome between insulin and metformin by 9 studies which involved 1135 GDM patients. In the pairwise meta-analysis, we observed that metformin had lower gestational weight gain compared with insulin (MD, 1.29; 95\% CI, 0.40 to $2.19 ; P<0.001)$. However, there was significant heterogeneity between these studies $\left(P<0.001, I^{2}=84 \%\right)$. Gestational weight gain was reported as an outcome between insulin and glyburide by 3 studies which included 523 GDM patients. There was no significant heterogeneity between these studies $\left(P=0.41, I^{2}=0 \%\right)$. However, there was no significant statistical difference between the insulin and glyburide groups in terms of gestational weight gain (MD, 0.66 ; 95\% CI, -0.36 to $1.69 ; P=0.20$ ). Three studies involving $376 \mathrm{GDM}$ patients reported the gestational weight gain between glyburide and metformin. There was no significant heterogeneity between these studies $\left(P=0.45, I^{2}=0 \%\right)$.
In the pairwise meta-analysis, we observed that metformin had lower gestational weight gain compared with glyburide (MD, $1.67 ; 95 \% \mathrm{CI}, 0.26$ to $3.07 ; P=0.02$ ).

3.3.7. Glycemic Control (the HbA1c\% at Delivery, Glycated Hemoglobin at Weeks 36-37). The HbA1c\% at delivery was included as an outcome between insulin and metformin by 5 studies which involved 932 GDM patients. There was no significant heterogeneity between these studies $(P=0.84$, $\left.I^{2}=0 \%\right)$. In the pairwise meta-analysis, we observed that metformin had the lower $\mathrm{HbA1c} \%$ at weeks 36-37 compared with insulin (MD, 0.18 ; 95\% CI, 0.07 to $0.29 ; P<0.01$ ).

Glycated hemoglobin at weeks 36-37 was reported as an outcome between metformin and insulin by 6 studies which involved 1539 GDM patients. There was significant heterogeneity between these studies $\left(P<0.0001, I^{2}=84 \%\right)$. The pooled result showed no significant statistical difference between the metformin and insulin groups in terms of glycated hemoglobin at weeks $36-37$ (MD, 0.06; 95\% CI, -0.05 to $0.18 ; P=0.29$ ).

3.3.8. Fasting Blood Glucose (FBG). FBG was included as an outcome between metformin and insulin by 9 studies which involved 2641 GDM patients. There was significant heterogeneity between these studies $\left(P<0.00001, I^{2}=95 \%\right)$. The pooled result showed no significant statistical difference between the metformin and insulin groups in terms of FBG (MD, $0.64 ; 95 \% \mathrm{CI},-1.56$ to $2.84 ; P=0.87$ ). Three studies involving 582 GDM patients reported the FBG between insulin and glyburide. There was significant heterogeneity between these studies $\left(P<0.001, I^{2}=86 \%\right)$. The pooled result showed no significant statistical difference between the insulin and glyburide in terms of FBG (MD, 2.54; 95\% CI, -4.98 to $10.06 ; P=0.51$ ).

3.3.9. Two-Hour Postprandial Glucose (2HPG). $2 \mathrm{HPG}$ was included as an outcome between insulin and metformin by 6 studies which involved 2315 GDM patients. There was significant heterogeneity between these studies $(P<0.001$, $\left.I^{2}=92 \%\right)$. The pooled result showed no significant statistical difference between the insulin and metformin groups in terms of $2 \mathrm{HPG}$ (MD, $1.61 ; 95 \% \mathrm{CI},-0.34$ to $3.56 ; P=0.11$ ).

3.3.10. NICU Admission. NICU admission was included as an outcome between metformin and insulin by 14 studies which involved 2402 GDM patients. There was no significant heterogeneity between these studies $\left(P=0.60, I^{2}=0 \%\right)$. In the pairwise meta-analysis, we observed that metformin had lower incidence of NICU admission compared with insulin (RR, 0.75; 95\% CI, 0.64 to $0.87 ; P<0.001$ ). NICU admission was reported as an outcome between glyburide and insulin by 7 studies which included 1751 GDM patients. There was no significant heterogeneity between these studies $\left(P=0.65, I^{2}\right.$ $=0 \%)$. However, there was no significant statistical difference between the glyburide and insulin groups in terms of NICU admission (OR, $0.94 ; 95 \% \mathrm{CI}, 0.58$ to $1.51 ; P=0.78$ ). Three studies involving 421 GDM patients focused on the incidence of NICU admission between glyburide and metformin. There was no significant heterogeneity between these 
studies $\left(P=0.42, I^{2}=0 \%\right)$. However, there was no significant statistical difference between the glyburide and metformin groups in terms of NICU admission (RR, 0.55; 95\% CI, 0.26 to $1.16 ; P=0.12$ ).

3.3.11. Need for Neonatal Dextrose. Need for neonatal dextrose was included as an outcome between metformin and insulin by 3 studies which involved 255 GDM patients. There was no significant heterogeneity between these studies $\left(P=0.45, I^{2}=0 \%\right)$. However, there was no significant statistical difference between the metformin and insulin groups in terms of need for neonatal dextrose (RR, 1.06; 95\% CI, 0.67 to $1.68 ; P=0.81$ ).

3.3.12. Neonatal Hypocalcemia. Neonatal hypocalcemia was included as an outcome between glyburide and insulin by 3 studies which involved 749 GDM patients. There was no significant heterogeneity between these studies $\left(P=0.26, I^{2}=20 \%\right)$. However, there was no significant statistical difference between the glyburide and insulin groups in terms of neonatal hypocalcemia (OR, 0.53; 95\% CI, 0.11 to $2.63 ; P=0.43$ ).

3.3.13. Congenital Anomaly. Congenital anomaly was included as an outcome between metformin and insulin by 6 studies which involved 839 GDM patients. There was no significant heterogeneity between these studies $(P=0.31$, $\left.I^{2}=17 \%\right)$. However, there was no significant statistical difference between the metformin and insulin groups in terms of congenital anomaly (OR, $0.78 ; 95 \%$ CI, 0.29 to $2.11 ; P=0.63)$. Seven studies involving 1049 GDM patients focused on the incidence of the congenital anomaly between glyburide and insulin. There was no significant heterogeneity between these studies $\left(P=0.87, I^{2}=0 \%\right)$. However, there was no significant statistical difference between the glyburide and insulin groups in terms of congenital anomaly (RR, 1.06; 95\% CI, 0.73 to $1.54 ; P=0.76$ ).

3.3.14. Neonatal Hypoglycemia. Neonatal hypoglycemia was included as an outcome between metformin and insulin by 15 studies which involved 2755 GDM patients. There was no significant heterogeneity between these studies $(P=0.66$, $\left.I^{2}=0 \%\right)$. In the pairwise meta-analysis, we observed that metformin had lower incidence of neonatal hypoglycemia compared with insulin (RR, 0.57; 95\% CI, 0.49 to 0.66 ; $P<0.00001)$. Neonatal hypoglycemia was reported as an outcome between glyburide and insulin by 12 studies which included 2406 GDM patients. There was no significant heterogeneity between these studies $\left(P=0.21, I^{2}=25 \%\right)$. In the pairwise meta-analysis, we observed that glyburide had higher incidence of neonatal hypoglycemia compared with insulin (RR, 1.76; 95\% CI, 1.32 to 2.36; $P<0.001$ ). Five studies involving $684 \mathrm{GDM}$ patients focused on the incidence of neonatal hypoglycemia between glyburide and metformin. There was no significant heterogeneity between these studies $\left(P=0.09, I^{2}=50 \%\right)$. However, there was no significant statistical difference between the glyburide and metformin groups in terms of neonatal hypoglycemia (RR, $1.03 ; 95 \% \mathrm{CI}, 0.39$ to 2.74; $P=0.95)$.
3.3.15. Birth Injury. Birth injury was included as an outcome between metformin and insulin by 7 studies which involved 1769 GDM patients. There was significant heterogeneity between these studies $\left(P=0.11, I^{2}=55 \%\right)$. Also, there was no significant statistical difference between the metformin and insulin groups in terms of birth injury (OR, 1.12; 95\% CI, 0.66 to $1.89 ; P=0.67$ ).

3.3.16. Sepsis. Sepsis was included as an outcome between metformin and insulin by 4 studies which involved 1167 GDM patients. There was significant heterogeneity between these studies ( $\left.P=0.11, I^{2}=55 \%\right)$. Also, there was no significant statistical difference between the metformin and insulin groups in terms of birth injury (OR, 1.12; 95\% CI, 0.66 to $1.89 ; P=0.67)$.

3.3.17. Five-Minute Apgar Score. The 5-minute Apgar score was included as an outcome between insulin and metformin and by 8 studies which involved 1059 GDM patients. There was significant heterogeneity between these studies $\left(P<0.001, I^{2}=79 \%\right)$. The pooled result showed no significant statistical difference between the insulin and metformin groups in terms of the 5-minute Apgar score (RR, $0.05 ; 95 \% \mathrm{CI},-0.19$ to $0.28 ; P=0.68$ ).

3.3.18. Five-Minute Apgar Score <7. The 5-minute Apgar score $<7$ was included as an outcome between metformin and insulin by 5 studies which involved 1585 GDM patients. There was no significant heterogeneity between these studies $\left(P=0.88, I^{2}=0 \%\right)$. However, there was no significant statistical difference between the metformin and insulin groups in terms of the 5-minute Apgar score $<7$ (OR, 1.29; 95\% CI, 0.70 to $2.38 ; P=0.42$ ). The 5 -minute Apgar score $<7$ was reported as an outcome between glyburide and metformin by 3 studies which included 325 GDM patients. There was no significant heterogeneity between these studies $(P=0.30$, $\left.I^{2}=7 \%\right)$. However, there was no significant statistical difference between the glyburide and metformin groups in terms of the 5-minute Apgar score $<7$ (RR, 0.88; 95\% CI, 0.13 to $5.89 ; P=0.90)$.

3.3.19. Macrosomia. Macrosomia was included as an outcome between metformin and insulin by 13 studies which involved $2331 \mathrm{GDM}$ patients. There was no significant heterogeneity between these studies $\left(P=0.38, I^{2}=6 \%\right)$. In the pairwise meta-analysis, we observed that metformin had lower incidence of macrosomia compared with insulin (RR, $0.68 ; 95 \%$ CI, 0.55 to $0.86 ; P<0.05$ ). Nine studies involving 2227 GDM patients focused on the incidence of macrosomia between glyburide and insulin. There was significant heterogeneity between these studies $(P=0.02$, $\left.I^{2}=56 \%\right)$. The pooled result showed no significant statistical difference in terms of macrosomia (RR, $1.46 ; 95 \% \mathrm{CI}, 0.78$ to $2.75 ; P=0.24$ ). Four studies involving $484 \mathrm{GDM}$ patients focused on the incidence of macrosomia between glyburide and metformin. There was no significant heterogeneity between these studies $\left(P=0.39, I^{2}=1 \%\right)$. However, there was no significant statistical difference between the glyburide 
and metformin groups in terms of macrosomia (OR, 1.45; $95 \% \mathrm{CI}, 0.63$ to $3.37 ; P=0.39$ ).

3.3.20. Respiratory Distress Syndrome (RDS). RDS was included as an outcome between metformin and insulin by 12 studies which involved 2172 GDM patients. There was significant heterogeneity between these studies $(P=0.01$, $\left.I^{2}=55 \%\right)$. Also, there was no significant statistical difference between the metformin and insulin groups in terms of RDS (OR, 1.03; 95\% CI, 0.68 to $1.56 ; P=0.88$ ).

3.3.21. Shoulder Dystocia. Shoulder dystocia was included as an outcome between metformin and insulin by 6 studies which involved 625 GDM patients. There was no significant heterogeneity between these studies $\left(P=0.15, I^{2}=40 \%\right)$. However, there was no significant difference between the metformin and insulin groups in terms of shoulder dystocia (OR, 1.33; 95\% CI, 0.36 to $4.94 ; P=0.67$ ).

3.3.22. Neonatal Jaundice/Hyperbilirubinemia. Neonatal jaundice/hyperbilirubinemia was included as an outcome between metformin and insulin by 13 studies which involved 2378 GDM patients. There was no significant heterogeneity between these studies $\left(P=0.03, I^{2}=47 \%\right)$. However, there was no significant statistical difference between the metformin and insulin groups in terms of neonatal jaundice/hyperbilirubinemia (RR, 1.07; 95\% CI, 0.94 to 1.23; $P=0.31)$. Neonatal jaundice/hyperbilirubinemia was reported as an outcome between glyburide and insulin by 5 studies which included 1618 GDM patients. There was no significant heterogeneity between these studies $(P=0.43$, $\left.I^{2}=0 \%\right)$. However, there was no significant statistical difference between the glyburide and insulin groups in terms of neonatal jaundice/hyperbilirubinemia (RR, 1.09; 95\% CI, 0.84 to $1.41 ; P=0.52$ ).

3.3.23. Large for Gestational Age (>90th Percentile). LGA ( $>90$ th percentile) was included as an outcome between metformin and insulin by 13 studies which involved 2812 GDM patients. There was no significant heterogeneity between these studies $\left(P=0.51, I^{2}=0 \%\right)$. However, there was no significant statistical difference between the metformin and insulin groups in terms of LGA (RR, $0.87 ; 95 \% \mathrm{CI}, 0.74$ to $1.02 ; P=$ $0.09)$. Five studies involving 1006 GDM patients focused on the incidence of LGA between glyburide and insulin. There was significant heterogeneity between these studies $(P=0.02$, $\left.I^{2}=66 \%\right)$. The pooled result showed no significant statistical difference between the glyburide and insulin groups in terms of LGA (RR, 1.66 ; $95 \%$ CI, 0.83 to $3.31 ; P=0.15$ ). Four studies involving $376 \mathrm{GDM}$ patients focused on the incidence of LGA between glyburide and metformin. There was significant heterogeneity between these studies $\left(P=0.03, I^{2}=72 \%\right)$. The pooled result showed no significant statistical difference between the glyburide and metformin groups in terms of LGA (RR, $0.67 ; 95 \% \mathrm{CI}, 0.25$ to $1.76 ; P=0.41)$.

3.3.24. Small for Gestational Age (<10th Percentile). SGA $(<10$ th percentile) was included as an outcome between metformin and insulin by 12 studies which involved 2833 GDM patients. There was no significant heterogeneity between these studies $\left(P=0.04, I^{2}=47 \%\right)$. However, there was no significant statistical difference between the metformin and insulin groups in terms of SGA (RR, 1.06; $95 \% \mathrm{CI}, 0.82$ to $1.37 ; P=0.65)$.

3.3.25. Transient Tachypnea. Transient tachypnea was included as an outcome between metformin and insulin by 4 studies which involved 1104 GDM patients. There was no significant heterogeneity between these studies $(P=0.92$, $\left.I^{2}=0 \%\right)$. However, there was no significant statistical difference between the metformin and insulin groups in terms of transient tachypnea (OR, $0.76 ; 95 \% \mathrm{CI}, 0.36$ to $1.57 ; P=0.45$ ).

3.3.26. Birth Weight. Birth weight was included as an outcome between metformin and insulin by 16 studies which involved 2853 GDM patients. There was significant heterogeneity between these studies $\left(P<0.001, I^{2}=83 \%\right)$. However, in the pairwise meta-analysis, we observed that metformin had lower birth weight compared with insulin (MD, 114.48; 95\% CI, 37.32 to $191.64 ; P<0.01) .10$ studies involving 1980 GDM patients reported the birth weight between glyburide and insulin. There was significant heterogeneity between these studies $\left(P<0.001, I^{2}=86 \%\right)$. The pooled result showed no significant statistical difference between the glyburide and insulin groups in terms of birth weight (MD, 62.58; 95\% CI, -55.98 to $181.14 ; P=0.30$ ). Six studies involving 707 GDM patients reported the birth weight between glyburide and metformin. There was significant heterogeneity between these studies $\left(P=0.03, I^{2}=61 \%\right)$. The pooled result showed no significant statistical difference between the glyburide and metformin groups in terms of birth weight (MD, 92.64; 95\% CI, -10.60 to $195.88 ; P=0.08$ ).

3.3.27. Umbilical Artery $p H$. Umbilical artery $\mathrm{pH}$ was included as an outcome between metformin and insulin by 6 studies which involved 961 GDM patients. There was no significant heterogeneity between these studies $\left(P=0.98, I^{2}=0 \%\right)$. However, there was no significant statistical difference between the metformin and insulin groups in terms of umbilical artery $\mathrm{pH}(\mathrm{MD}, 0.00 ; 95 \% \mathrm{CI},-0.01$ to $0.01 ; P=0.64)$.

3.3.28. Neonatal Blood Glucose. Neonatal blood glucose was included as an outcome between metformin and insulin by 3 studies which involved 384 GDM patients. There was no significant heterogeneity between these studies $(P=0.17$, $\left.I^{2}=44 \%\right)$. In the pairwise meta-analysis, we observed that insulin had lower neonatal blood glucose compared with metformin (MD, 2.95; 95\% CI, 0.63 to 5.26 ; $P<0.05$ ).

\section{Discussion}

The purpose of this meta-analysis was to evaluate the efficacy and safety of three drugs (metformin, glyburide, and insulin) for GDM. Several maternal and neonatal outcomes were assessed.

Our meta-analysis, in accordance with the result of previous review [74], suggested that metformin could be a safe and effective treatment for GDM. There was no significant difference between metformin and insulin in terms of glycemic control; the other comparative two groups did not reveal a 
significant difference either. Differently [74], metformin had a lower risk of preeclampsia compared with insulin groups (RR, $0.57 ; 95 \%$ CI, 0.45 to $0.72 ; P<0.001$ ). To the outcomes of neonatal blood glucose and birth weight, metformin had a lower increase when compared with insulin (MD, 2.95; 95\% CI, 0.63 to $5.26 ; P<0.05 ; \mathrm{MD}, 114.48 ; 95 \% \mathrm{CI}, 37.32$ to 191.64; $P<0.01$; respectively). Mothers receiving metformin had a lower incidence of maternal hypoglycemia (RR, 0.28; 95\% CI, 0.10 to $0.75 ; P=0.05$ ), gestational hypertension (RR, $0.56 ; 95 \% \mathrm{CI}, 0.36$ to $0.87 ; P<0.01$ ), and induction of labor (RR, 0.85 ; $95 \% \mathrm{CI}, 0.74$ to $0.99 ; P<0.05$ ) than those in the insulin group. Compared with metformin, insulin had a significant increase in the gestational age at delivery (MD, 0.23 ; 95\% CI, 0.12 to $0.34 ; P<0.001$ ); likewise, insulin had a risk of macrosomia (RR, 0.68 ; $95 \%$ CI, 0.55 to 0.86 ; $P<0.05)$. Risks of NICU admission and neonatal hypoglycemia were lower in the metformin group and reached a statistically significant level when compared with insulin (RR, 0.75; 95\% CI, 0.64 to $0.87 ; P<0.001$; RR, $0.57 ; 95 \% \mathrm{CI}, 0.49$ to $0.66 ; P<0.001$; respectively). We observed no statistically significant difference in other outcomes. However, the latest study [75] showed that with dietary and lifestyle advice started at 10-20 weeks' gestation when metformin was given to overweight or obese pregnant women, metformin cannot improve pregnancy and birth outcomes. Statistically significant heterogeneity prevented analyses of the outcomes of gestational weight gain and birth weight. The outcomes for other differences between the two groups remained nonsignificant.

Metformin had higher gestational weight gain compared with glyburide (MD, 1.67; 95\% CI, 0.26 to $3.07 ; P<0.05$ ). For the rest of the outcomes, no significant difference was noticed between the two groups. Of the two groups between glyburide and insulin, glyburide had a higher risk of neonatal hypoglycemia compared with insulin (RR, 1.76; 95\% CI, 1.32 to 2.36; $P<0.001$ ), which is the same as the study [23]. In the study [22], significant differences for outcomes in between glyburide and insulin were obtained in birth weight (MD, 109; $95 \%$ CI, 35.9 to $181 ; P<0.01$ ), macrosomia (RR, 2.62; 95\% CI, 1.35 to $5.08 ; P<0.01$ ), and neonatal hypoglycemia (RR, 2.04; 95\% CI, 1.30 to $3.20 ; P<0.01$ ), which are different from our study, but in our meta-analysis, there are more subjects. No other significant difference was noticed between the two groups.

In the meta-analysis [28], glyburide ranked the worst with the highest incidence of macrosomia, preeclampsia, hyperbilirubinemia, neonatal hypoglycemia, preterm birth, and low birth weight; metformin (plus insulin when required) has the lowest risk of macrosomia, pregnancy hypertension, LGA, RDS, preterm birth, and low birth weight. Besides, insulin had the highest incidence of NICU admission.

Our findings, which are based on more recent studies, except for preterm birth, are in accordance with the results of previous meta-analyses $[6,16,17,23,73,75-81]$. Gui et al.'s study [73] including 3 RCTs revealed that metformin had a significantly higher risk than insulin in terms of preterm birth, the incidence of preterm birth was significantly higher in the metformin group than in the insulin group (OR, 1.74; 95\% CI, 1.13 to $2.68 ; P=0.01$ ); also, there was no significant heterogeneity between these studies $\left(P=0.84, I^{2}=0 \%\right)$. In our meta-analysis, which has 11 RCTs, in addition, we calculated RR to analyze the data. Su and Wang's study [81] including 6 RCTs revealed that metformin had a higher incidence of preterm birth compared with insulin (RR, 1.56 ; $95 \% \mathrm{CI}, 1.06$ to $2.30 ; P=0.01$ ). In this meta-analysis, there is no forest plot about each evaluation index and the search flow diagram; besides, our meta-analysis has increased sample size. Therefore, our results are more reliable. In contrast to other meta-analyses, in the process of searching, we searched five databases and used the keywords with metformin, glyburide, insulin, GDM, and RCT, to assure more and complete articles being included. 12 primary outcomes and 24 secondary outcomes were detected and analyzed; our meta-analysis is more detailed compared with others. In addition, there were two forms of outcomes, forest plots and funnel plots.

A major strength of this study was the comprehensive coverage of the literature achieved by including the most up-to-date review on the topic and including 41 RCTs in the medical literature and the comparison of three drugs (metformin, glyburide, and insulin) in the treatment of GDM patients. Moreover, it is evident from the bias summary that 2 studies were at high risk of two types of bias.

However, there were several limitations to the metaanalysis that deserve comment. First, some of the outcomes were only included by a few studies, and there have been insufficient power to detect important differences between treatment groups. Second, definitions for GDM and some outcomes (e.g., gestational hypertension, neonatal hypoglycemia, and macrosomia) were either not defined by some studies or the definitions varied between studies. Third, none of these studies evaluated long-term maternal and neonatal outcomes. Moreover, the different gestational ages at enrollment might also result in heterogeneity in gestational weight gain.

\section{Conclusions}

In summary, based on the short-term data available, metformin could be a safe and effective treatment for GDM. However, clinicians should pay attention to the relative lack of long-term offspring data with GDM patients treated with metformin. Compared with insulin, glyburide had a higher increase of neonatal hypoglycemia. The other use of glyburide in pregnancy for GDM women appears to be unclear. Clinicians should weigh in practice the condition of patients when selecting different GDM treatment strategy. Further studies with larger sample sizes are required to confirm the long-term maternal and neonatal outcomes in the metformin-treated GDM patients for the safety of metformin as a universal treatment in GDM patients and to reassess the efficacy and safety of glyburide in the treatment of GDM patients.

\section{Appendix}

\section{A. Maternal and Neonatal Outcomes (Forest Plots)}

\author{
A.1. Maternal Outcomes (Figures 4-10)
}




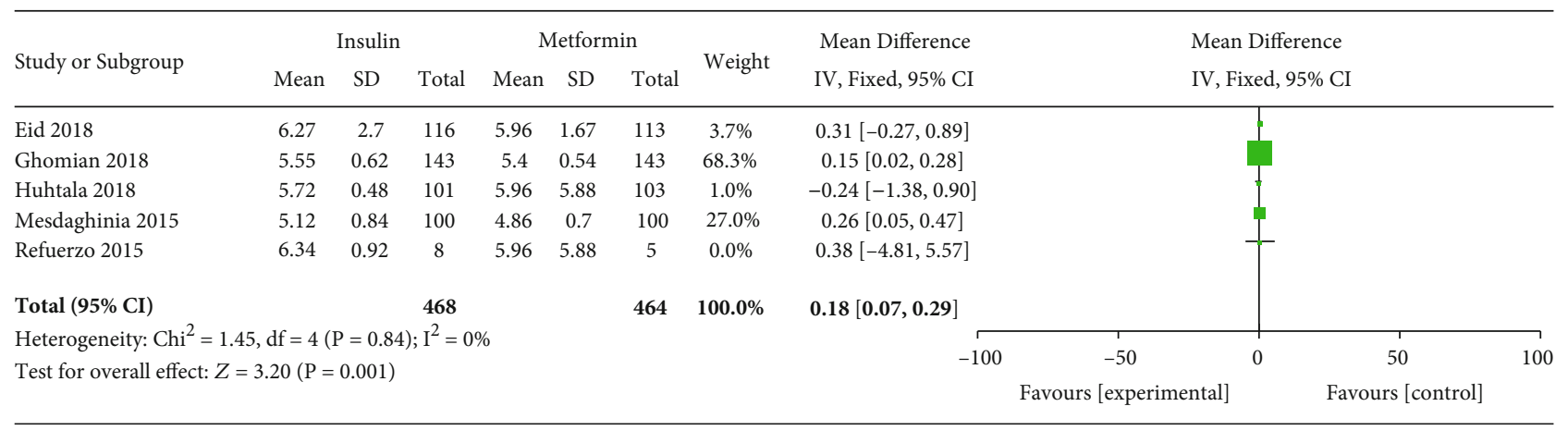

Figure 4: HbAlc\% at delivery.

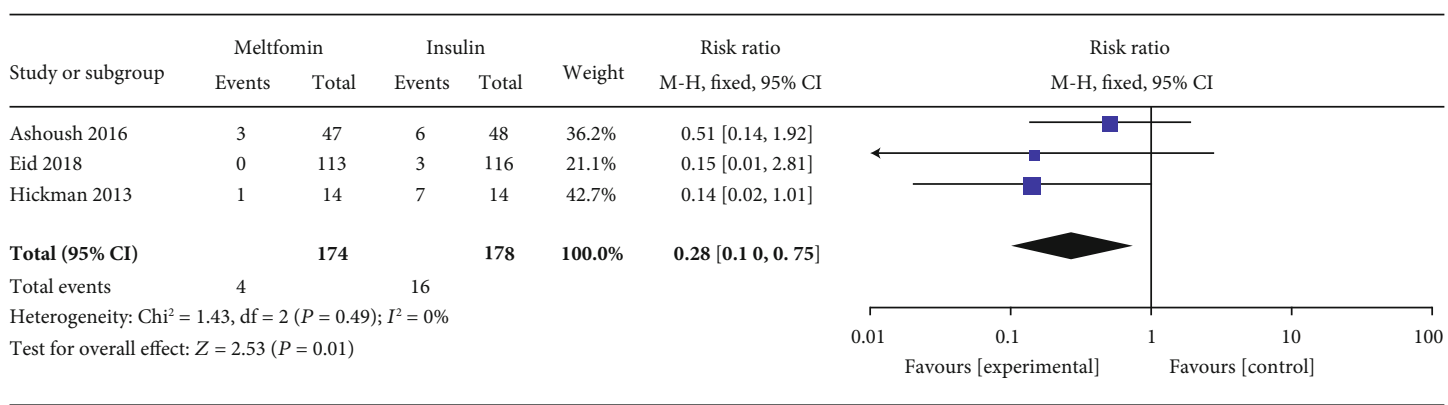

FIgURE 5: Maternal hypoglycemia.

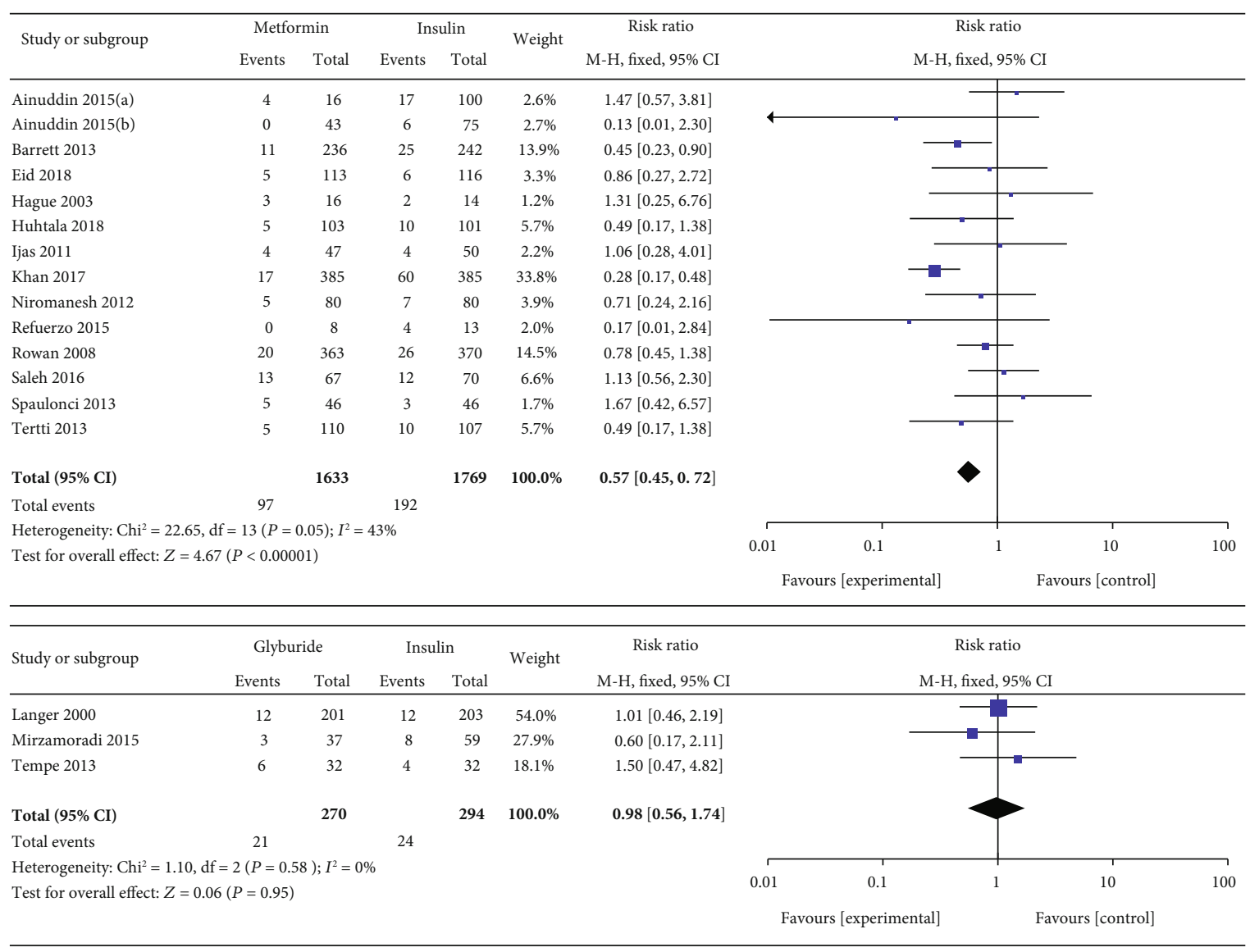




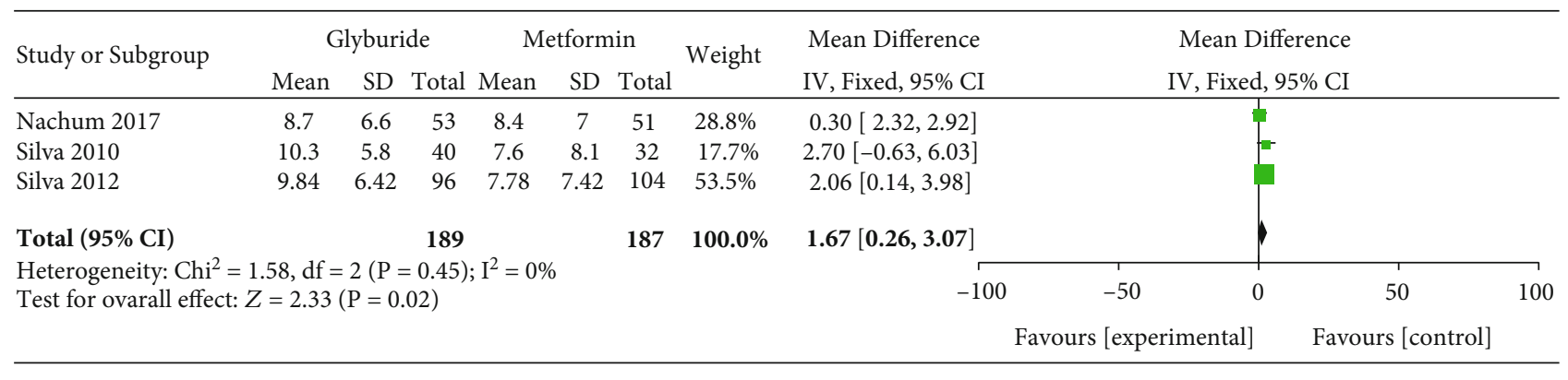

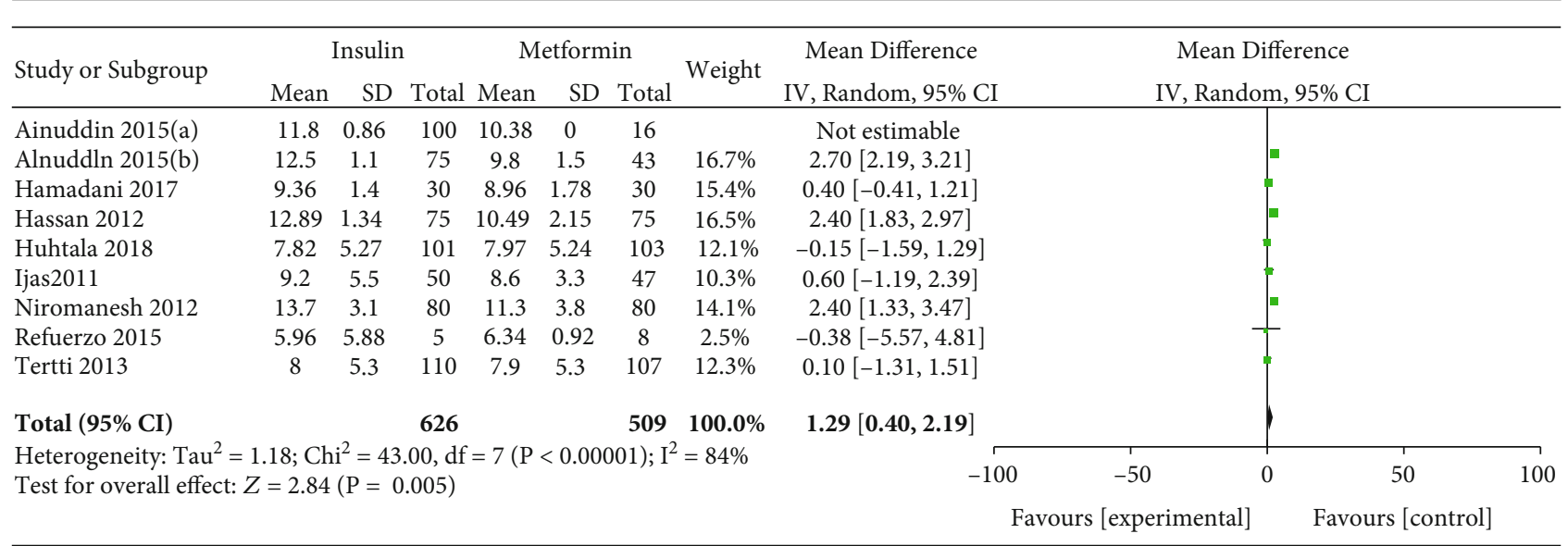

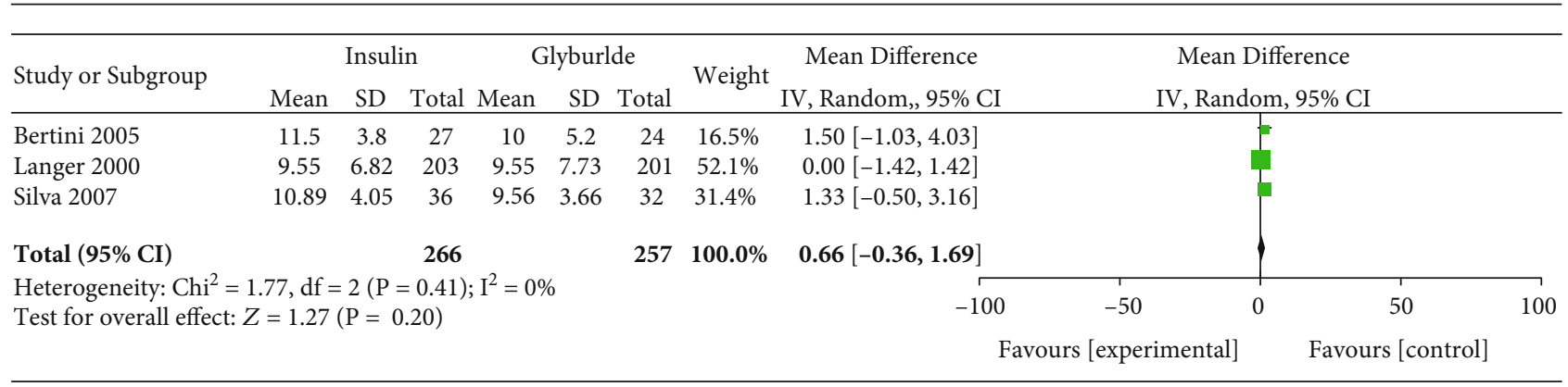

FIGURE 7: Gestational weight gain. 


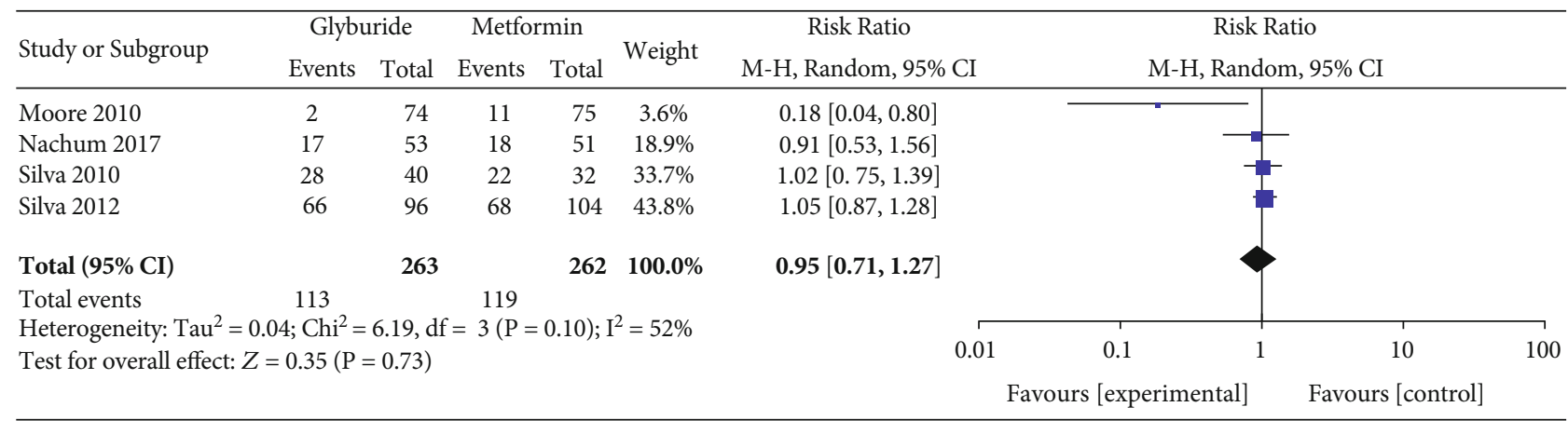

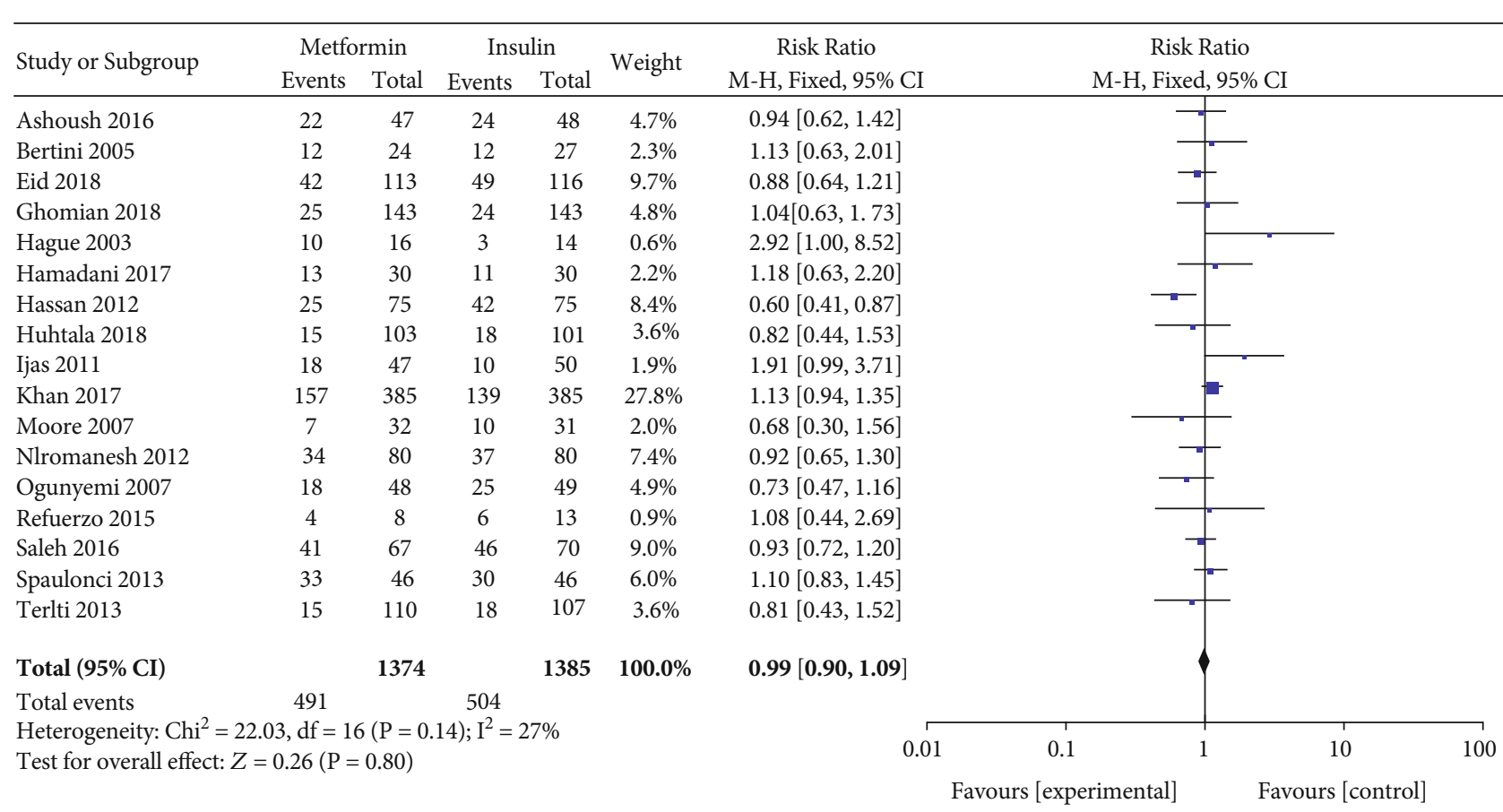

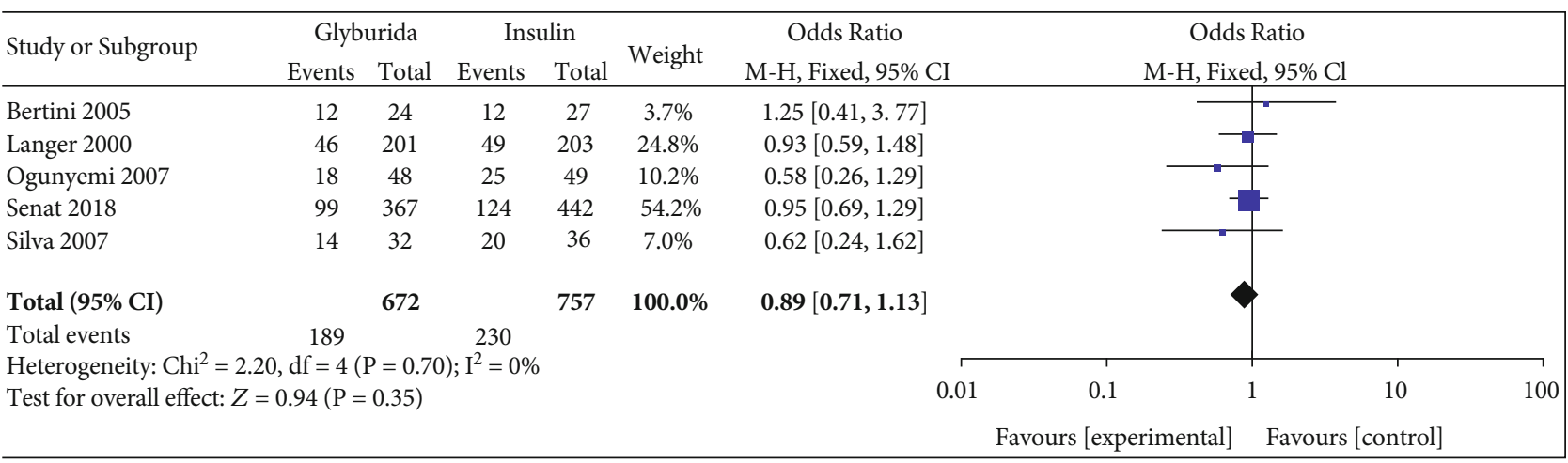

FIGURE 8: Cesarean section. 


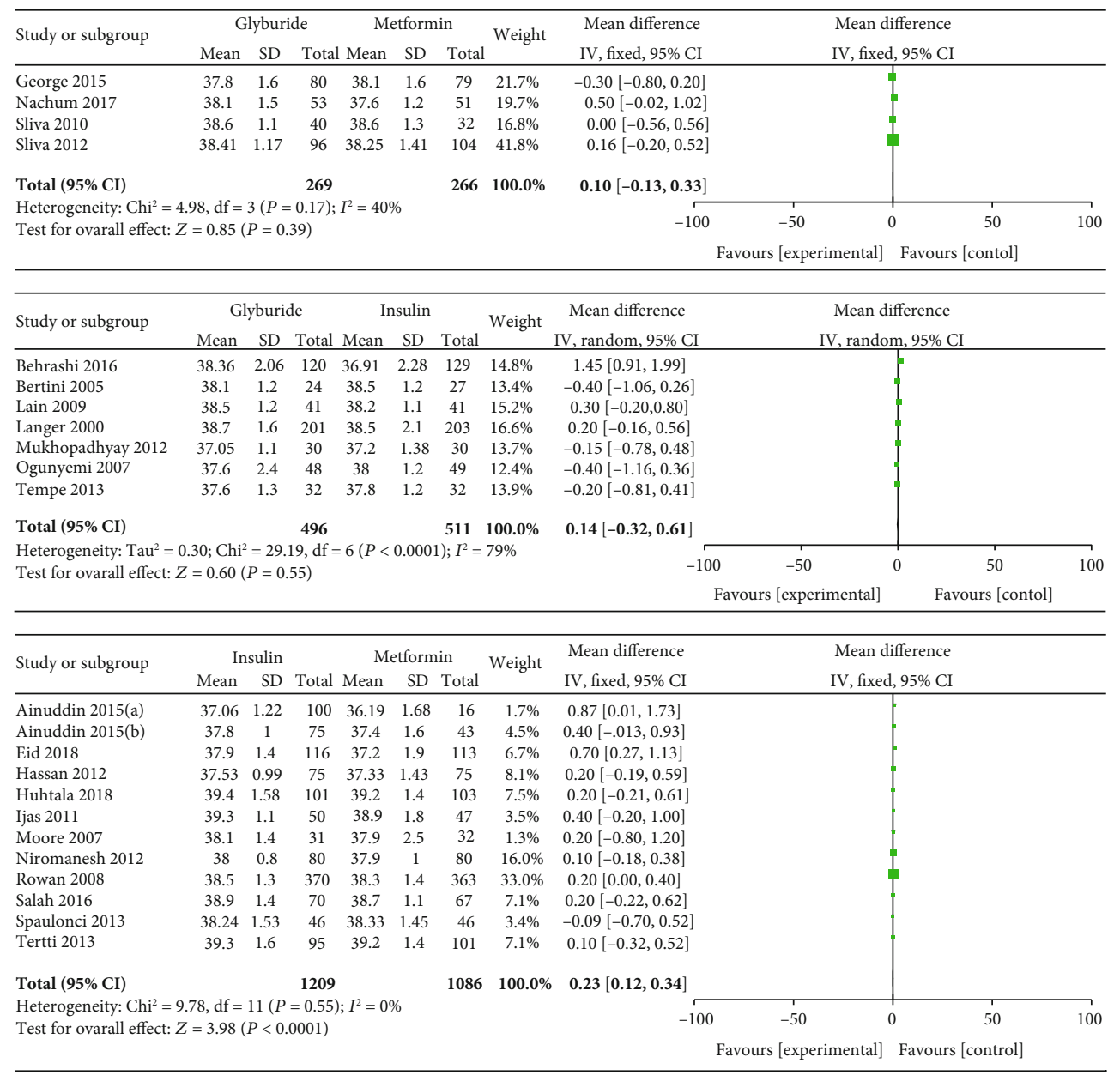

Figure 9: Gestational age at delivery.

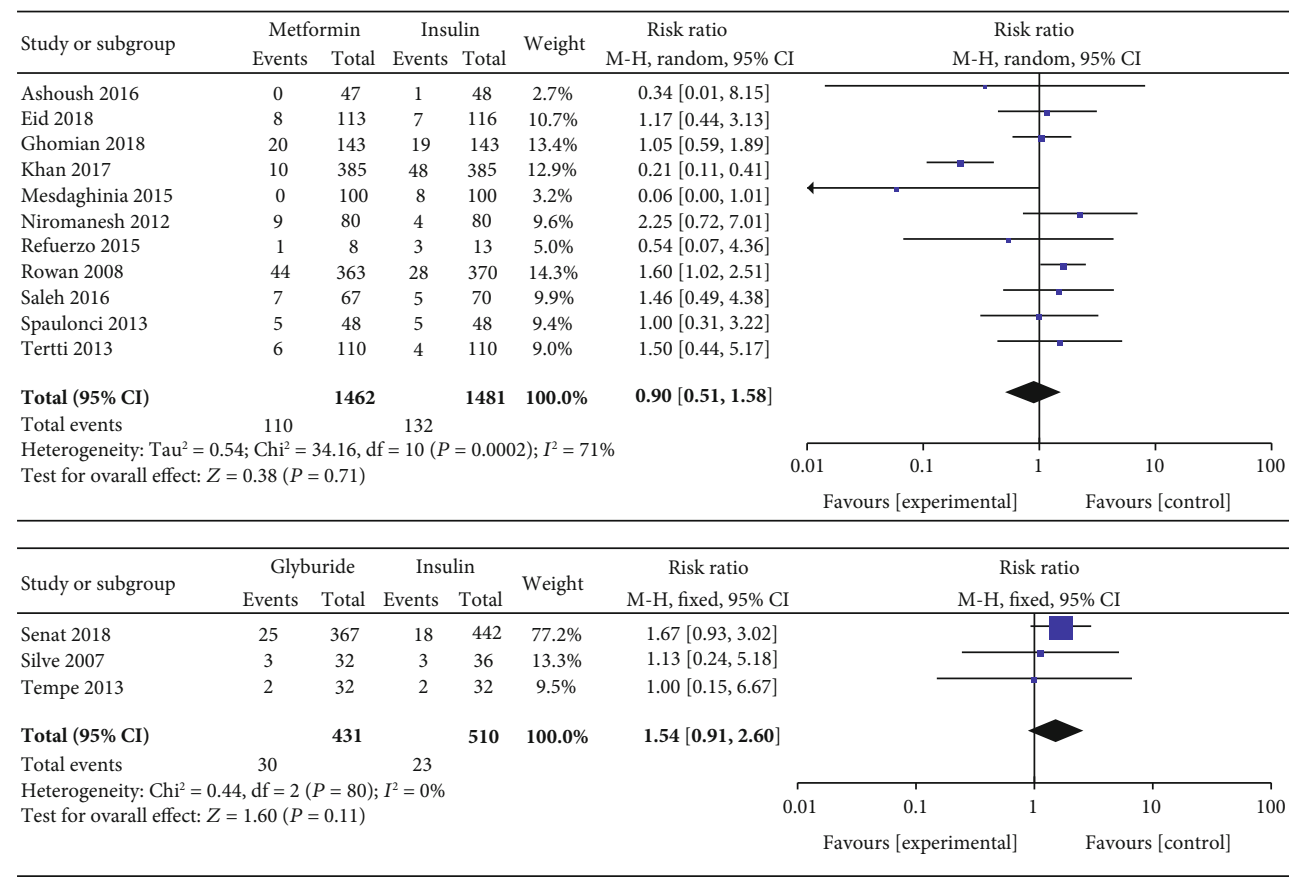




\section{A.2. Neonatal Outcomes (Figures 11-16)}

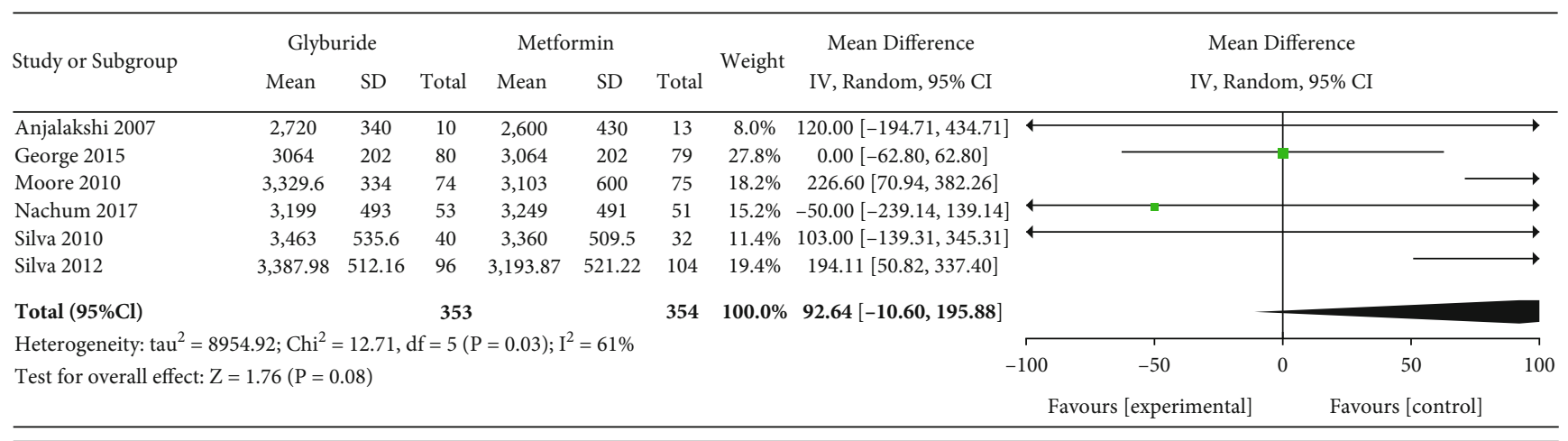

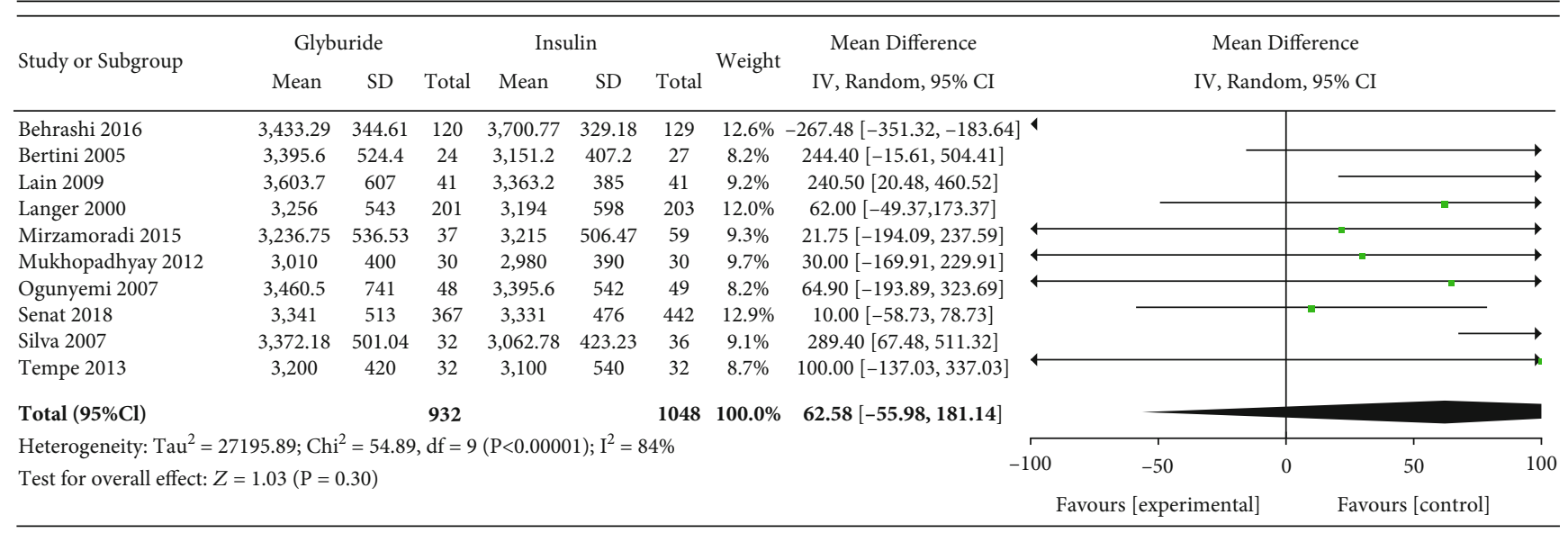

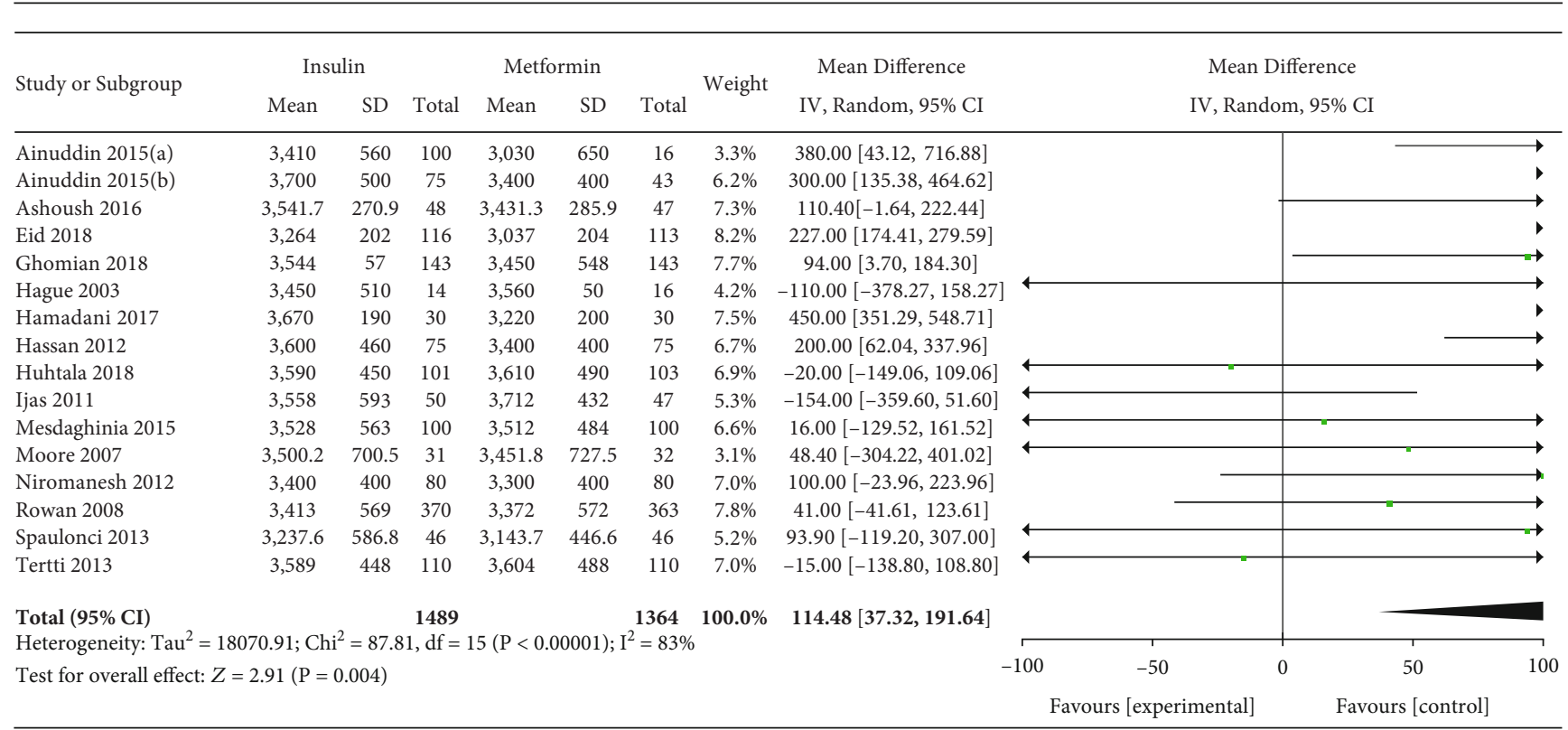

Figure 11: Birth weight. 


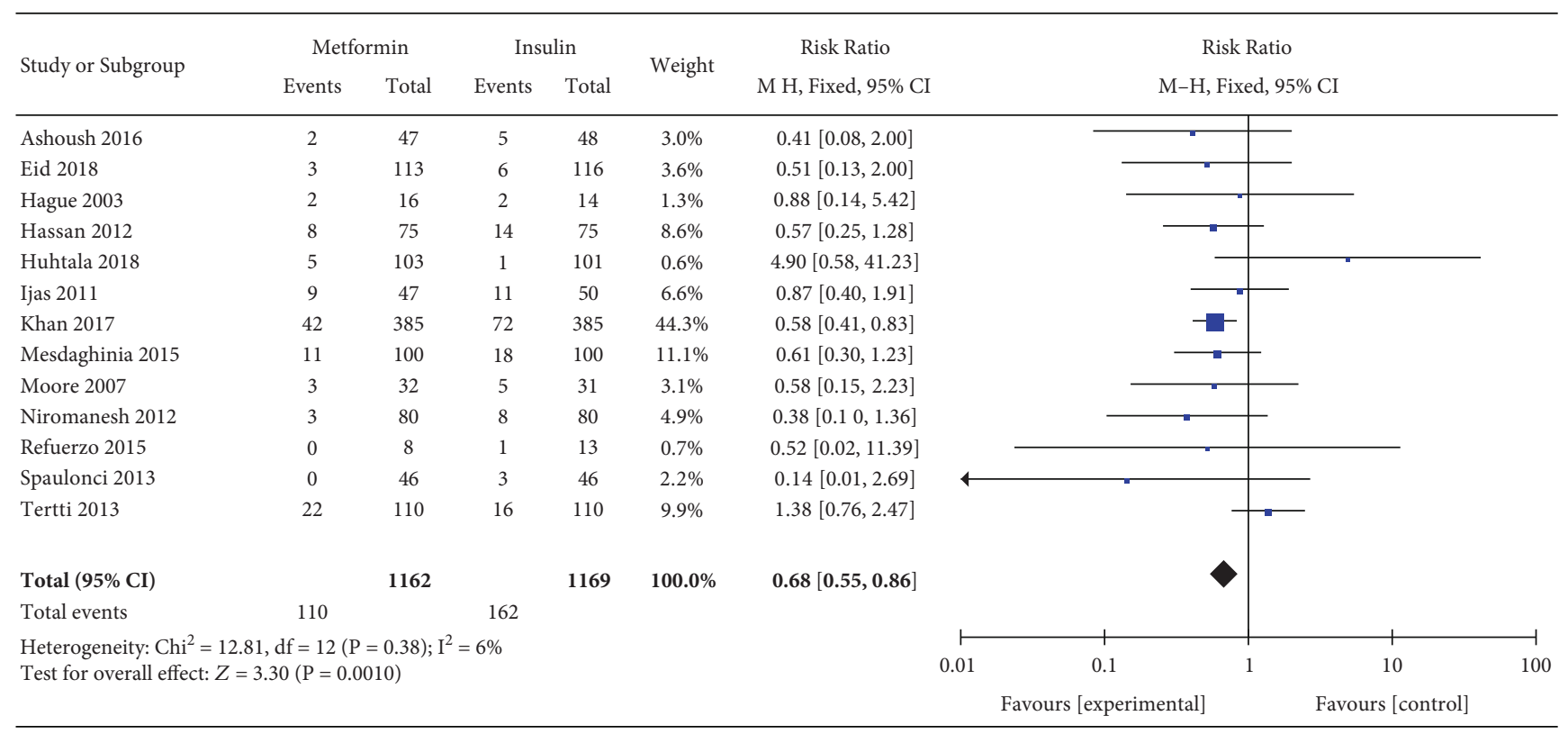

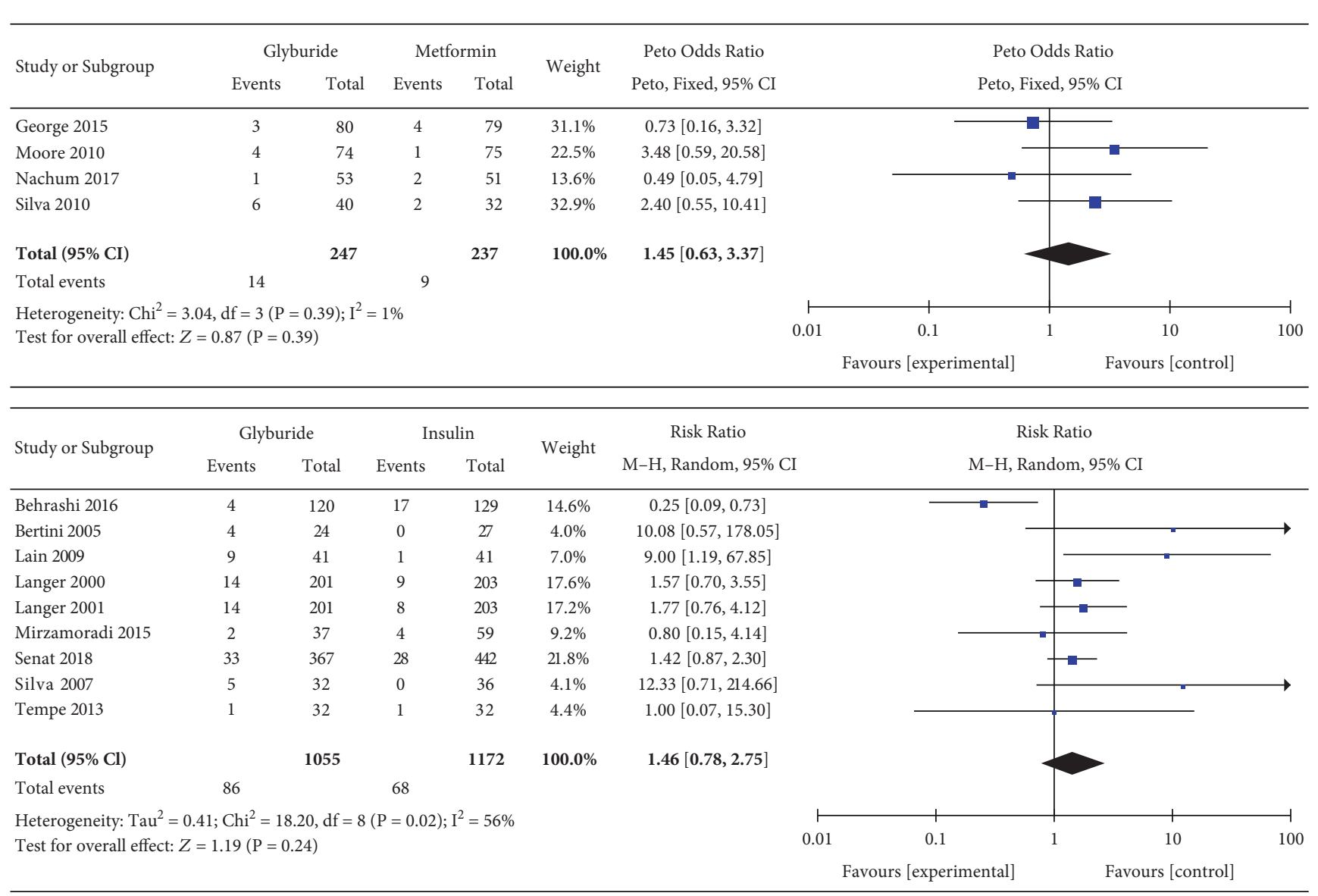

Figure 12: Macrosomia. 


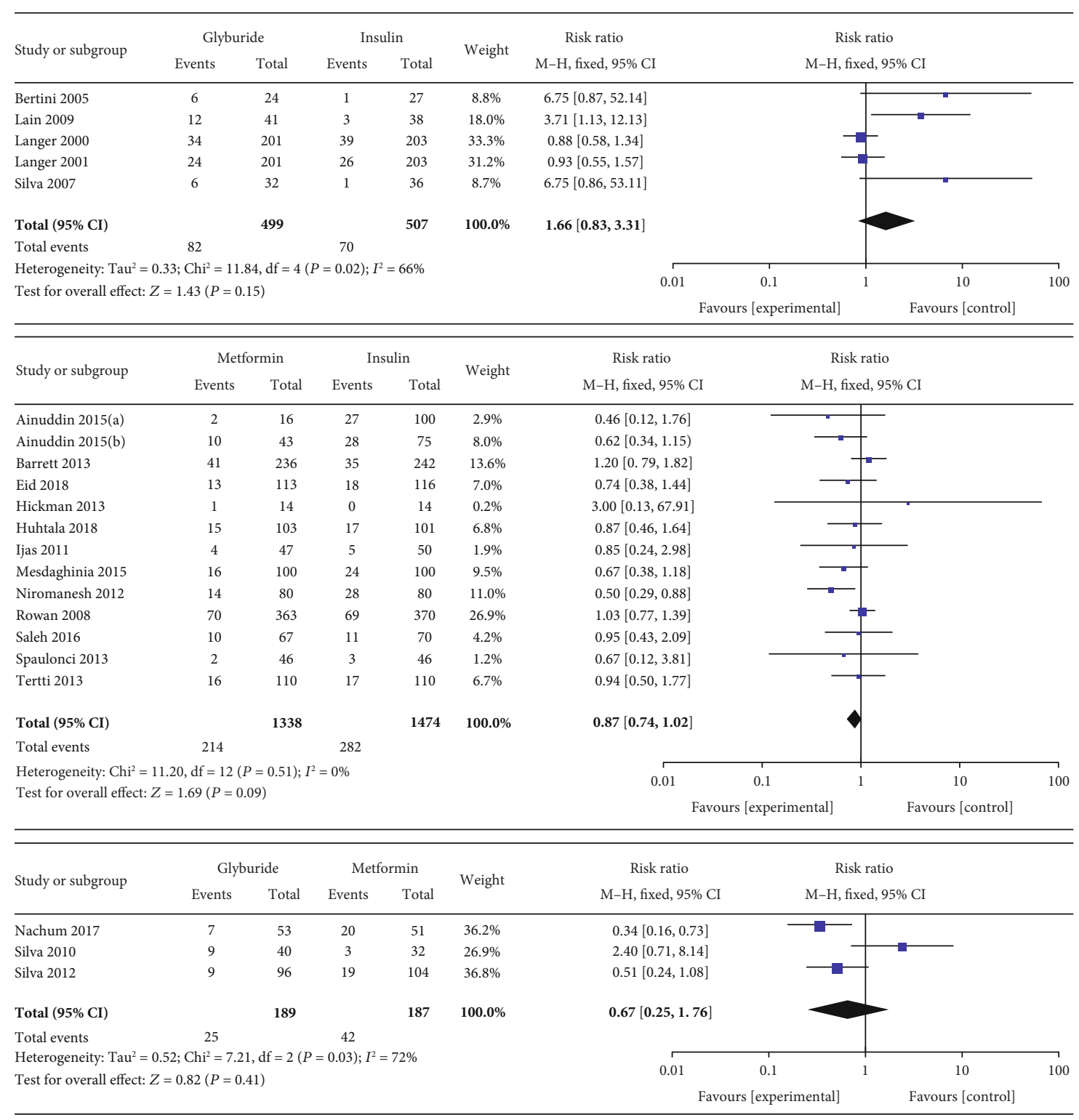

Figure 13: Large for gestational age.

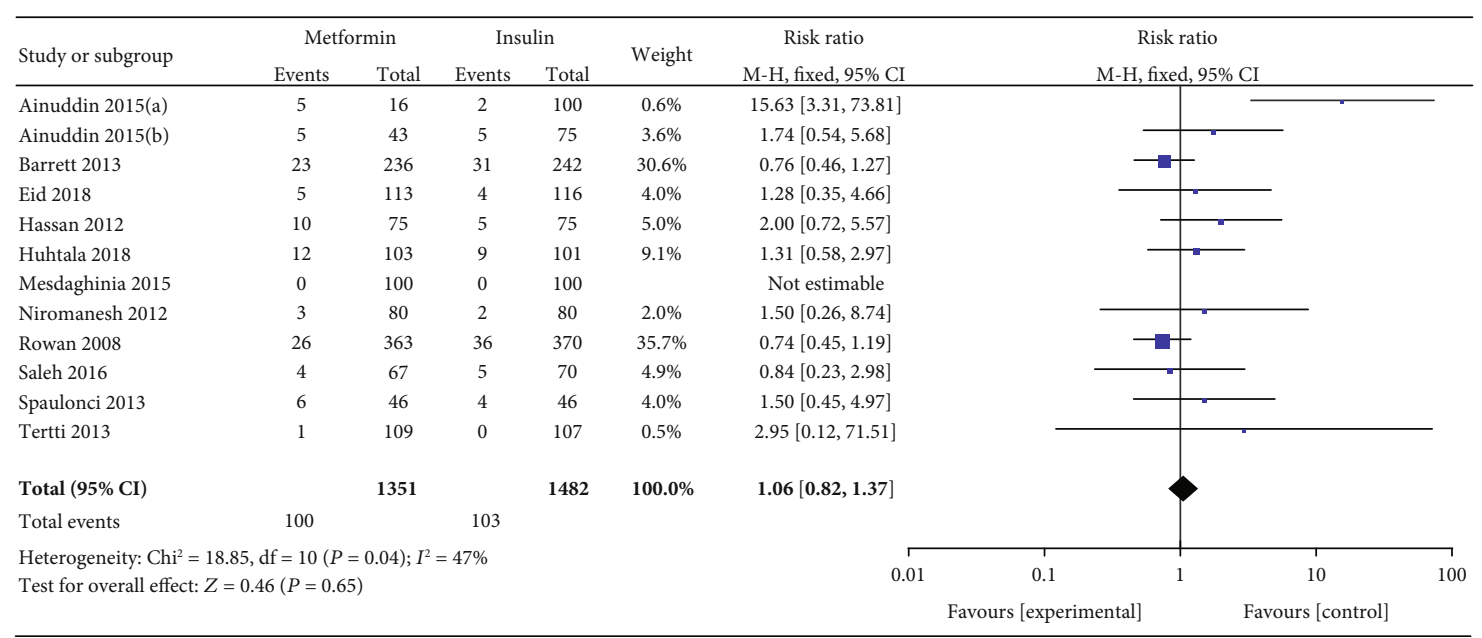

FIGURE 14: Small for gestational age. 


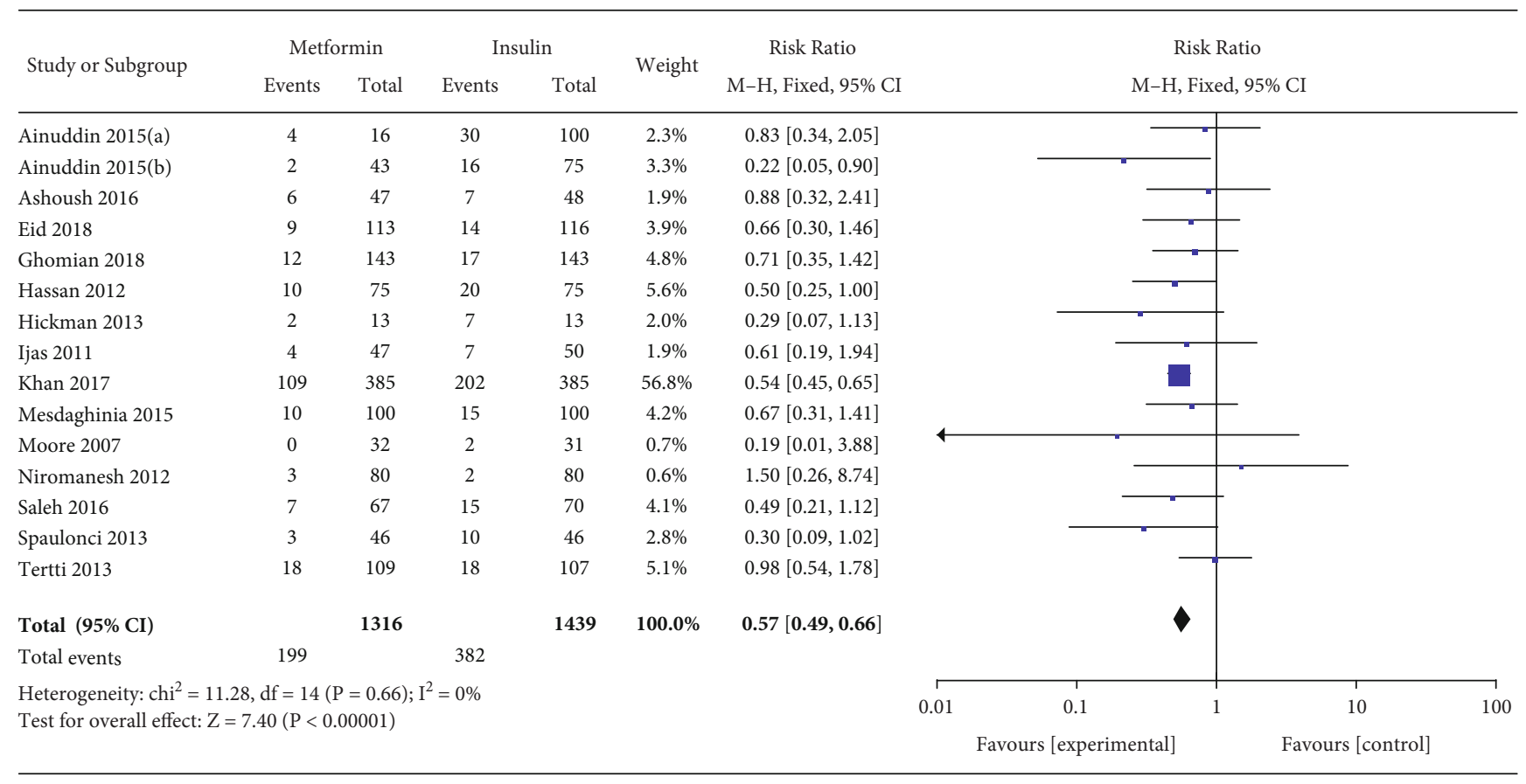

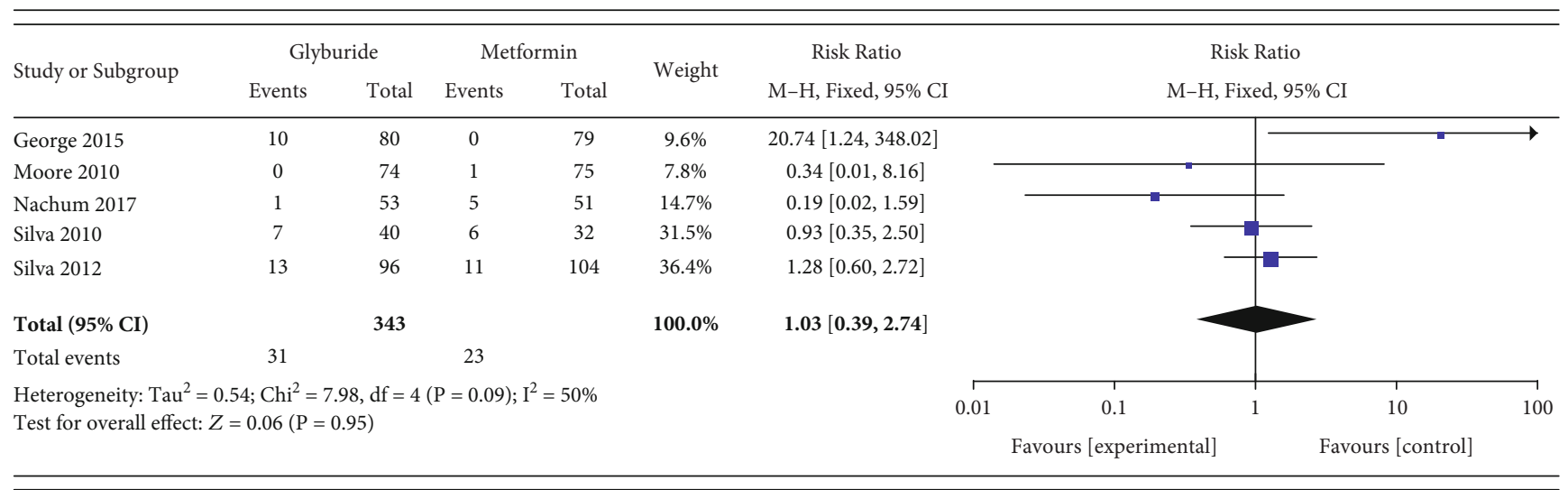

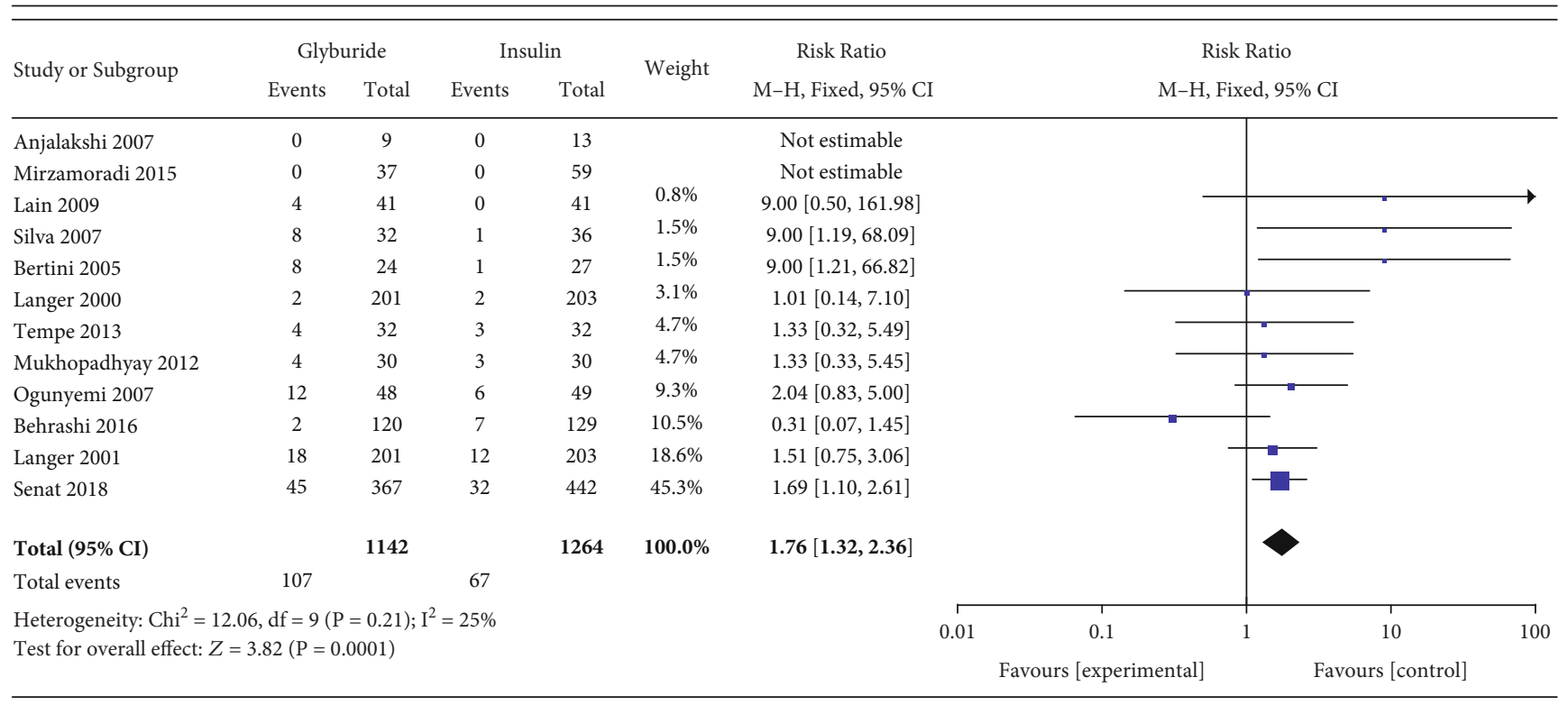




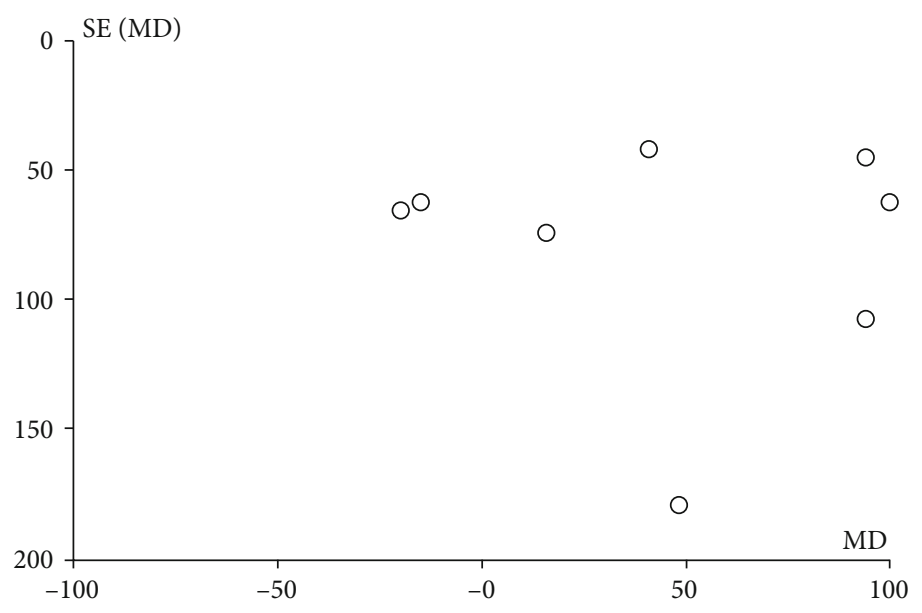

FIGURE 16: Funnel plots: birth weight (insulin vs. metformin).

\section{Conflicts of Interest}

The authors declare that there is no conflict of interest regarding the publication of this paper.

\section{Authors' Contributions}

Lanlan Guo had full access to all of the data in the study and accepts responsibility for the data management and accuracy of the data analysis. Study concept and design were the responsibility of Jing $\mathrm{Ma}$ and Jia Tang. Acquisition, analysis, and interpretation of the data were done by Lanlan Guo, Dingyao $\mathrm{Hu}$, Wei Zhang, and Xue Zhao. Drafting of the manuscript was done by Lanlan Guo and Jia Tang. Critical revision of the manuscript for important intellectual content was the responsibility of Jing Ma and Jia Tang. All authors have read and approved the final version of the manuscript and agree to submit it for consideration for publication in the journal. Lanlan Guo and Jing Ma contributed equally to this study.

\section{Supplementary Materials}

According to the suggestion, when the number of events was too low $(P<0.05)$, Peto odds ratio was used to analyze the outcome index. As demonstrated, these ten outcome indexes included macrosomia (glyburide vs. metformin), NICU admission (glyburide vs. insulin), respiratory distress syndrome (RDS) (glyburide vs. insulin), birth injury (metformin vs. insulin), 5-minute Apgar score $<7$ (metformin vs. insulin), congenital anomaly (metformin vs. insulin), neonatal hypocalcemia (glyburide vs. insulin), sepsis (metformin vs. insulin), shoulder dystocia (metformin vs. insulin), and transient tachypnea (metformin vs. insulin). As we can see, in the outcome indexes, all of them did not show a statistically significant difference. (Supplementary Materials)

\section{References}

[1] Y. Zhu and C. Zhang, "Prevalence of gestational diabetes and risk of progression to type 2 diabetes: a global perspective," Current Diabetes Reports, vol. 16, no. 1, p. 7, 2016.
[2] B. E. Metzger, S. G. Gabbe, B. Persson et al., "International association of diabetes and pregnancy study groups recommendations on the diagnosis and classification of hyperglycemia in pregnancy," Diabetes Care, vol. 33, no. 3, pp. 676-682, 2010.

[3] B. E. Metzger, L. P. Lowe, A. R. Dyer et al., "Hyperglycemia and adverse pregnancy outcomes," The New England Journal of Medicine, vol. 358, no. 19, pp. 1991-2002, 2008.

[4] W. L. Lowe Jr., D. M. Scholtens, L. P. Lowe et al., “Association of gestational diabetes with maternal disorders of glucose metabolism and childhood adiposity," JAMA, vol. 320 , no. 10 , pp. 1005-1016, 2018.

[5] M. B. Landon, C. Y. Spong, E. Thom et al., "A multicenter, randomized trial of treatment for mild gestational diabetes," The New England Journal of Medicine, vol. 361, no. 14, pp. 1339-1348, 2009.

[6] J. Brown, L. Grzeskowiak, K. Williamson, M. R. Downie, and C. A. Crowther, "Insulin for the treatment of women with gestational diabetes," Cochrane Database of Systematic Reviews, no. 11, article CD012037, 2017.

[7] M. J. Nahuis, E. J. Oude Lohuis, N. Bayram et al., "Pregnancy complications and metabolic disease in women with clomiphene citrate-resistant anovulation randomized to receive laparoscopic electrocautery of the ovaries or ovulation induction with gonadotropins: a 10-year follow-up," Fertility and Sterility, vol. 101, no. 1, pp. 270-274, 2014.

[8] American Diabetes Association, "Management of diabetes in pregnancy," Diabetes Care, vol. 40, Supplement 1, pp. S114S119, 2017.

[9] J. Girard, "The inhibitory effects of insulin on hepatic glucose production are both direct and indirect," Diabetes, vol. 55, Supplement 2, pp. S65-S69, 2006.

[10] E. K. Berggren and K. A. Boggess, "Oral agents for the management of gestational diabetes," Clinical Obstetrics and Gynecology, vol. 56, no. 4, pp. 827-836, 2013.

[11] N. Kavitha, S. De, and S. Kanagasabai, "Oral hypoglycemic agents in pregnancy: an update," The Journal of Obstetrics and Gynecology of India, vol. 63, no. 2, pp. 82-87, 2013.

[12] N. Magon and V. Seshiah, "Gestational diabetes mellitus: noninsulin management," Indian Journal of Endocrinology and Metabolism, vol. 15, no. 4, pp. 284-293, 2011.

[13] R. J. Norman, J. X. Wang, and W. Hague, "Should we continue or stop insulin sensitizing drugs during pregnancy?," Current 
Opinion in Obstetrics \& Gynecology, vol. 16, no. 3, pp. 245250, 2004.

[14] Y. F. Jiang, X. Y. Chen, T. Ding, X. F. Wang, Z. N. Zhu, and S. W. Su, "Comparative efficacy and safety of OADs in management of GDM: network meta-analysis of randomized controlled trials," The Journal of Clinical Endocrinology and Metabolism, vol. 100, no. 5, pp. 2071-2080, 2015.

[15] S. Lv, J. Wang, and Y. Xu, "Safety of insulin analogs during pregnancy: a meta-analysis," Archives of Gynecology and Obstetrics, vol. 292, no. 4, pp. 749-756, 2015.

[16] J. S. Refuerzo, "Oral hypoglycemic agents in pregnancy," Obstetrics and Gynecology Clinics of North America, vol. 38, no. 2, pp. 227-234, 2011, ix.

[17] X. M. Zhen, X. Li, and C. Chen, "Metformin versus insulin for gestational diabetes: the reporting of ethnicity and a metaanalysis combining English and Chinese literatures," Obesity Medicine, vol. 11, pp. 48-58, 2018.

[18] A. J. Mulherin, A. H. Oh, H. Kim, A. Grieco, L. M. Lauffer, and P. L. Brubaker, "Mechanisms underlying metformin-induced secretion of glucagon-like peptide-1 from the intestinal L cell," Endocrinology, vol. 152, no. 12, pp. 4610-4619, 2011.

[19] W. Nicholson, S. Bolen, C. T. Witkop, D. Neale, L. Wilson, and E. Bass, "Benefits and risks of oral diabetes agents compared with insulin in women with gestational diabetes: a systematic review," Obstetrics and Gynecology, vol. 113, no. 1, pp. 193205, 2009.

[20] American Diabetes Association, "Management of diabetes in pregnancy," Diabetes Care, vol. 39, Supplement 1, pp. S94S98, 2016.

[21] J. A. Rowan, W. M. Hague, W. Gao, M. R. Battin, M. P. Moore, and MiG Trial Investigators, "Metformin versus insulin for the treatment of gestational diabetes," The New England Journal of Medicine, vol. 358, no. 19, pp. 2003-2015, 2008.

[22] M. Balsells, A. Garcia-Patterson, I. Sola, M. Roque, I. Gich, and R. Corcoy, "Glibenclamide, metformin, and insulin for the treatment of gestational diabetes: a systematic review and meta-analysis," BMJ, vol. 350, no. 14, 2015.

[23] R. Song, L. Chen, Y. Chen et al., "Comparison of glyburide and insulin in the management of gestational diabetes: a meta-analysis," PLoS One, vol. 12, no. 8, article e0182488, 2017.

[24] A. M. Bertini, J. C. Silva, W. Taborda et al., "Perinatal outcomes and the use of oral hypoglycemic agents," Journal of Perinatal Medicine, vol. 33, no. 6, pp. 519-523, 2005.

[25] M. Mirzamoradi, Z. Heidar, Z. Faalpoor, Z. Naeiji, and R. Jamali, "Comparison of glyburide and insulin in women with gestational diabetes mellitus and associated perinatal outcome: a randomized clinical trial," Acta Medica Iranica, vol. 53, no. 2, pp. 97-103, 2015.

[26] Y. C. Zeng, M. J. Li, Y. Chen et al., "The use of glyburide in the management of gestational diabetes mellitus: a meta-analysis," Advances in Medical Sciences, vol. 59, no. 1, pp. 95-101, 2014.

[27] W. C. Castillo, K. Boggess, T. Stürmer, M. A. Brookhart, D. K. Benjamin Jr., and M. J. Funk, "Association of adverse pregnancy outcomes with glyburide vs insulin in women with gestational diabetes," JAMA Pediatrics, vol. 169, no. 5, pp. 452-458, 2015.

[28] H. L. Liang, S. J. Ma, Y. N. Xiao, and H. Z. Tan, "Comparative efficacy and safety of oral antidiabetic drugs and insulin in treating gestational diabetes mellitus: an updated PRISMAcompliant network meta-analysis," Medicine, vol. 96, no. 38, article e7939, 2017.
[29] M. S. Huhtala, K. Tertti, O. Pellonperä, and T. Rönnemaa, "Amino acid profile in women with gestational diabetes mellitus treated with metformin or insulin," Diabetes Research and Clinical Practice, vol. 146, pp. 8-17, 2018.

[30] A. K. Singh and R. Singh, "Oral antidiabetic agents in gestational diabetes: a narrative review of current evidence," Expert Review of Endocrinology \& Metabolism, vol. 10, no. 2, pp. 211$225,2015$.

[31] O. Langer, D. L. Conway, M. D. Berkus, E. M. J. Xenakis, and O. Gonzales, "A comparison of glyburide and insulin in women with gestational diabetes mellitus," The New England Journal of Medicine, vol. 343, no. 16, pp. 1134-1138, 2000.

[32] W. T. Cefalu, E. G. Berg, M. Saraco, M. P. Petersen, S. Uelmen, and S. Robinson, "Management of diabetes in pregnancy: standards of medical care in diabetes-2019," Diabetes Care, vol. 42, Supplement 1, pp. S165-S172, 2019.

[33] Committee on Practice Bulletins-Obstetrics, "ACOG Practice Bulletin No. 190: gestational diabetes mellitus," Obstetrics and Gynecology, vol. 131, no. 2, pp. e49-e64, 2018.

[34] H. Kleinwechter, U. Schäfer-Graf, C. Bührer et al., "Gestational diabetes mellitus (GDM) diagnosis, therapy and follow-up care: practice guideline of the German Diabetes Association (DDG) and the German Association for Gynaecology and Obstetrics (DGGG)," Experimental and Clinical Endocrinology \& Diabetes, vol. 122, no. 7, pp. 395-405, 2014.

[35] J. P. T. Higgins and S. Green, "Cochrane handbook for systematic reviews of interventions 5.1.0. The Cochrane Collaboration," 2011.

[36] D. Moher, A. Liberati, J. Tetzlaff, D. G. Altman, and The PRISMA Group, "Preferred reporting items for systematic reviews and meta-analyses: the PRISMA statement," PLoS Medicine, vol. 6, no. 7, article e1000097, 2009.

[37] A. Tempe and R. D. Mayanglambam, "Glyburide as treatment option for gestational diabetes mellitus," The Journal of Obstetrics and Gynaecology Research, vol. 39, no. 6, pp. 1147-1152, 2013.

[38] M. Behrashi, M. Samimi, T. Ghasemi, F. Saberi, and F. Atoof, "Comparison of glibenclamide and insulin on neonatal outcomes in pregnant women with gestational diabetes," International Journal of Preventive Medicine, vol. 7, no. 1, p. 88, 2016.

[39] R. M. A. Khan, A. Mukhtar, and A. Khawar, "Comparison of metformin with insulin in the management of gestational diabetes," Medical Forum Monthly, vol. 28, no. 11, pp. 105-109, 2017.

[40] A. George, J. E. Mathews, D. Sam et al., "Comparison of neonatal outcomes in women with gestational diabetes with moderate hyperglycaemia on metformin or glibenclamide - a randomised controlled trial," The Australian and New Zealand Journal of Obstetrics and Gynaecology, vol. 55, no. 1, pp. 47-52, 2015.

[41] E. Mesdaghinia, M. Samimi, Z. Homaei, F. Saberi, S. G. A. Moosavi, and M. Yaribakht, "Comparison of newborn outcomes in women with gestational diabetes mellitus treated with metformin or insulin: a randomised blinded trial," International Journal of Preventive Medicine, vol. 4, no. 3, pp. 327-333, 2013.

[42] W. M. Hague, P. M. Davoren, J. Oliver, and J. Rowan, "Contraindications to use of metformin - metformin may be useful in gestational diabetes," BMJ, vol. 326, no. 7392, p. 762, 2003.

[43] H. S. Saleh, W. A. Abdelsalam, H. E. Mowafy, and A. A. Abd ElHameid, "Could metformin manage gestational diabetes 
mellitus instead of insulin?," International Journal of Reproductive Medicine, vol. 2016, Article ID 3480629, 8 pages, 2016.

[44] M. V. Sénat, H. Affres, A. Letourneau et al., "Effect of glyburide vs subcutaneous insulin on perinatal complications among women with gestational diabetes a randomized clinical trial," JAMA, vol. 319, no. 17, pp. 1773-1780, 2018.

[45] N. Ghomian, S. H. M. Vahed, S. Firouz, M. A. Yaghoubi, M. Mohebbi, and A. Sahebkar, "The efficacy of metformin compared with insulin in regulating blood glucose levels during gestational diabetes mellitus: a randomized clinical trial," Journal of Cellular Physiology, vol. 234, no. 4, pp. 4695-4701, 2019.

[46] S. Waheed, F. P. Malik, and S. B. Mazhar, "Efficacy of metformin versus insulin in the management of pregnancy with diabetes," Journal of the College of Physicians and Surgeons Pakistan, vol. 23, no. 12, pp. 866-869, 2013.

[47] R. M. Reynolds, F. C. Denison, E. Juszczak et al., "Glibenclamide and metfoRmin versus stAndard care in gEstational diabeteS (GRACES): a feasibility open label randomised trial," BMC Pregnancy and Childbirth, vol. 17, no. 1, p. 316, 2017.

[48] Z. Nachum, N. Zafran, R. Salim et al., "Glyburide versus metformin and their combination for the treatment of gestational diabetes mellitus: a randomized controlled study," Diabetes Care, vol. 40, no. 3, pp. 332-337, 2017.

[49] E. A. Ryan, "Glyburide was as safe and effective as insulin in gestational diabetes," Evidence-Based Medicine, vol. 6, no. 3, p. 79, 2001.

[50] S. Ashoush, M. El-Said, H. Fathi, and M. Abdelnaby, "Identification of metformin poor responders, requiring supplemental insulin, during randomization of metformin versus insulin for the control of gestational diabetes mellitus," Journal of Obstetrics and Gynaecology Research, vol. 42, no. 6, pp. 640-647, 2016.

[51] S. R. Eid, R. S. I. Moustafa, M. M. Salah et al., "Is metformin a viable alternative to insulin in the treatment of gestational diabetes mellitus (GDM)? Comparison of maternal and neonatal outcomes," Egyptian Pediatric Association Gazette, vol. 66, no. 1, pp. 15-21, 2018.

[52] H. L. Barrett, K. L. Gatford, C. M. Houda et al., "Maternal and neonatal circulating markers of metabolic and cardiovascular risk in the metformin in gestational diabetes (mig) trial," Diabetes Care, vol. 36, no. 3, pp. 529-536, 2013.

[53] L. E. Moore, C. M. Briery, D. Clokey et al., "Metformin and insulin in the management of gestational diabetes mellitus preliminary results of a comparison," Journal of Reproductive Medicine, vol. 52, no. 11, pp. 1011-1015, 2007.

[54] J. C. Silva, C. Pacheco, J. Bizato, B. V. de Souza, T. E. Ribeiro, and A. M. Bertini, "Metformin compared with glyburide for the management of gestational diabetes," International Journal of Gynecology \& Obstetrics, vol. 111, no. 1, pp. 37-40, 2010.

[55] L. E. Moore, D. Clokey, V. J. Rappaport, and L. B. Curet, "Metformin compared with glyburide in gestational diabetes a randomized controlled trial," Obstetrics and Gynecology, vol. 115, no. 1, pp. 55-59, 2010.

[56] S. Niromanesh, A. Alavi, F. R. Sharbaf, N. Amjadi, S. Moosavi, and S. Akbari, "Metformin compared with insulin in the management of gestational diabetes mellitus: a randomized clinical trial," Diabetes Research and Clinical Practice, vol. 98, no. 3, pp. 422-429, 2012.

[57] M. A. Hickman, R. McBride, K. A. Boggess, and R. Strauss, "Metformin compared with insulin in the treatment of preg- nant women with overt diabetes: a randomized controlled trial," American Journal of Perinatology, vol. 30, no. 6, pp. 483-490, 2013.

[58] J. A. Hassan, N. Karim, and Z. Sheikh, "Metformin prevents macrosomia and neonatal morbidity in gestational diabetes," Pakistan Journal of Medical Sciences, vol. 28, no. 3, pp. 384389, 2012.

[59] H. Ijäs, M. Vääräsmäki, L. Morin-Papunen et al., "Metformin should be considered in the treatment of gestational diabetes: a prospective randomised study," BJOG-An International Journal of Obstetrics \& Gynaecology, vol. 118, no. 7, pp. 880885, 2011.

[60] J. A. Ainuddin, N. Karim, S. Zaheer, S. S. Ali, and A. A. Hasan, "Metformin treatment in type 2 diabetes in pregnancy: an active controlled, parallel-group, randomized, open label study in patients with type 2 diabetes in pregnancy," Journal of Diabetes Research, vol. 2015, Article ID 325851, 11 pages, 2015.

[61] T. Beyuo, S. A. Obed, K. K. Adjepong-Yamoah, K. A. Bugyei, S. A. Oppong, and K. Marfoh, "Metformin versus insulin in the management of pre-gestational diabetes mellitus in pregnancy and gestational diabetes mellitus at the Korle Bu Teaching Hospital: a randomized clinical trial," PLoS One, vol. 10, no. 5,2015 .

[62] A. Hamadani, S. Zahid, and Z. B. Butt, "Metformin versus insulin treatment in gestational diabetes in pregnancy and their effects on neonatal birthweight," Pakistan Journal of Medical and Health Sciences, vol. 11, no. 3, pp. 914-916, 2017.

[63] J. Ainuddin, N. Karim, A. A. Hasan, and S. A. Naqvi, "Metformin versus insulin treatment in gestational diabetes in pregnancy in a developing country. A randomized control trial," Diabetes Research and Clinical Practice, vol. 107, no. 2, pp. 290-299, 2015.

[64] K. Tertti, U. Ekblad, P. Koskinen, T. Vahlberg, and T. Ronnemaa, "Metformin vs. insulin in gestational diabetes. A randomized study characterizing metformin patients needing additional insulin," Diabetes Obesity \& Metabolism, vol. 15, no. 3, pp. 246-251, 2013.

[65] K. Y. Lain, M. J. Garabedian, A. Daftary, and A. Jeyabalan, "Neonatal adiposity following maternal treatment of gestational diabetes with glyburide compared with insulin," American Journal of Obstetrics and Gynecology, vol. 200, no. 5, pp. 501.e1-501.e6, 2009.

[66] P. Mukhopadhyay, T. S. Bag, A. Kyal, D. P. Saha, and N. Khalid, "Oral hypoglycemic glibenclamide: can it be a substitute to insulin in the management of gestational diabetes mellitus? A comparative study," Journal of SAFOG, vol. 4, no. 1, pp. 28-31, 2012.

[67] J. C. Silva, D. Fachin, M. L. Coral, and A. M. Bertini, "Perinatal impact of the use of metformin and glyburide for the treatment of gestational diabetes mellitus," Journal of Perinatal Medicine, vol. 40, no. 3, pp. 225-228, 2012.

[68] J. S. Refuerzo, R. Gowen, C. Pedroza, M. Hutchinson, S. C. Blackwell, and S. Ramin, "A pilot randomized, controlled trial of metformin versus insulin in women with type 2 diabetes mellitus during pregnancy," American Journal of Perinatology, vol. 30, no. 2, pp. 163-170, 2015.

[69] C. Anjalakshi, V. Balaji, M. S. Balaji, and V. Seshiah, "A prospective study comparing insulin and glibenclamide in gestational diabetes mellitus in Asian Indian women," Diabetes Research and Clinical Practice, vol. 76, no. 3, pp. 474-475, 2007. 
[70] C. P. Spaulonci, L. S. Bernardes, T. C. Trindade, M. Zugaib, and R. P. V. Francisco, "Randomized trial of metformin vs insulin in the management of gestational diabetes," American Journal of Obstetrics and Gynecology, vol. 209, no. 1, pp. 34.e1-34.e7, 2013.

[71] D. Ogunyemi, M. Jesse, and M. Davidson, "Comparison of glyburide versus insulin in management of gestational diabetes mellitus," Endocrine Practice, vol. 13, no. 4, pp. 427-428, 2007.

[72] J. C. Silva, A. M. Bertini, W. Taborda et al., "Glibenclamide in the treatment for gestational diabetes mellitus in a compared study to insulin," Arquivos Brasileiros de Endocrinologia e Metabologia, vol. 51, no. 4, pp. 541-546, 2007.

[73] J. Gui, Q. Liu, and L. Feng, "Metformin vs insulin in the management of gestational Diabetes: a meta-analysis," PLoS One, vol. 8, no. 5, article e64585, 2013.

[74] P. Kitwitee, S. Limwattananon, C. Limwattananon et al., "Metformin for the treatment of gestational diabetes: an updated meta-analysis," Diabetes Research and Clinical Practice, vol. 109, no. 3, pp. 521-532, 2015.

[75] J. M. Dodd, J. Louise, A. R. Deussen et al., "Effect of metformin in addition to dietary and lifestyle advice for pregnant women who are overweight or obese: the GRoW randomised, doubleblind, placebo-controlled trial," The Lancet Diabetes \& Endocrinology, vol. 7, no. 1, pp. 15-24, 2019.

[76] M. Amin, N. Suksomboon, N. Poolsup, and O. Malik, "Comparison of glyburide with metformin in treating gestational diabetes mellitus: a systematic review and meta-analysis," Clinical Drug Investigation, vol. 35, no. 6, pp. 343-351, 2015.

[77] N. Poolsup, N. Suksomboon, and M. Amin, "Efficacy and safety of oral antidiabetic drugs in comparison to insulin in treating gestational diabetes mellitus: a meta-analysis," PLoS One, vol. 9, no. 10, 2014.

[78] L. P. Zhao, X. Y. Sheng, S. Zhou et al., "Metformin versus insulin for gestational diabetes mellitus: a meta-analysis," British Journal of Clinical Pharmacology, vol. 80, no. 5, pp. 12241234, 2015.

[79] M. Balsells, A. Garcia-Patterson, I. Sola, M. Roque, I. Gich, and R. Corcoy, "Glibenclamide, metformin, and insulin for the treatment of gestational diabetes: a systematic review and meta-analysis," Obstetrical \& Gynecological Survey, vol. 70, no. 5, pp. 305-307, 2015.

[80] Y. Feng and H. Yang, "Metformin - a potentially effective drug for gestational diabetes mellitus: a systematic review and metaanalysis," The Journal of Maternal-Fetal \& Neonatal Medicine, vol. 30, no. 15, pp. 1874-1881, 2017.

[81] D. F. Su and X. Y. Wang, "Metformin vs insulin in the management of gestational diabetes: a systematic review and metaanalysis," Diabetes Research and Clinical Practice, vol. 104, no. 3, pp. 353-357, 2014. 


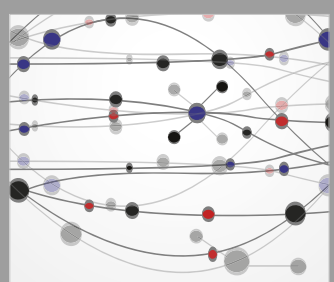

The Scientific World Journal
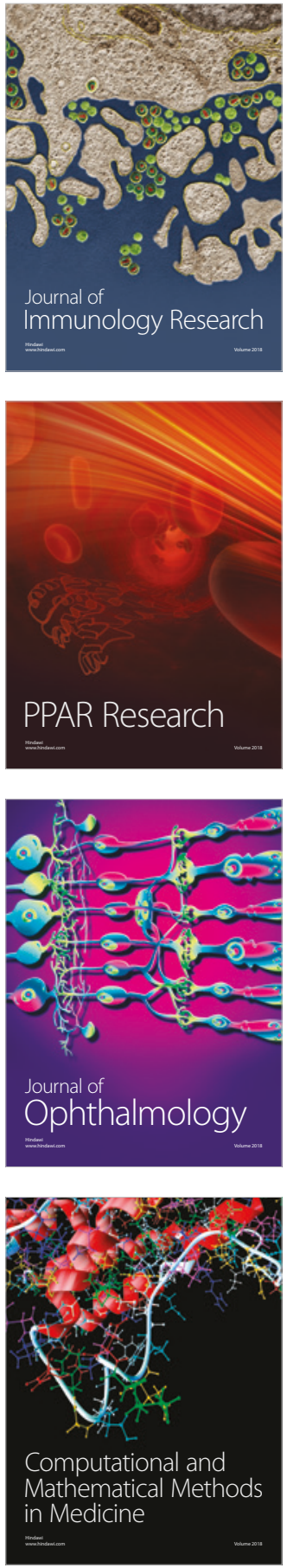

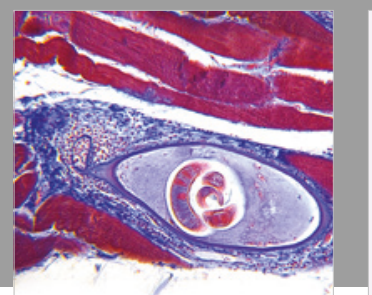

Gastroenterology Research and Practice

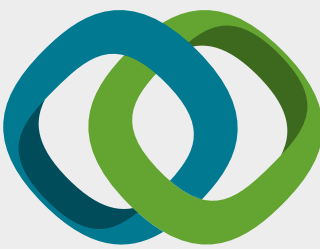

\section{Hindawi}

Submit your manuscripts at

www.hindawi.com
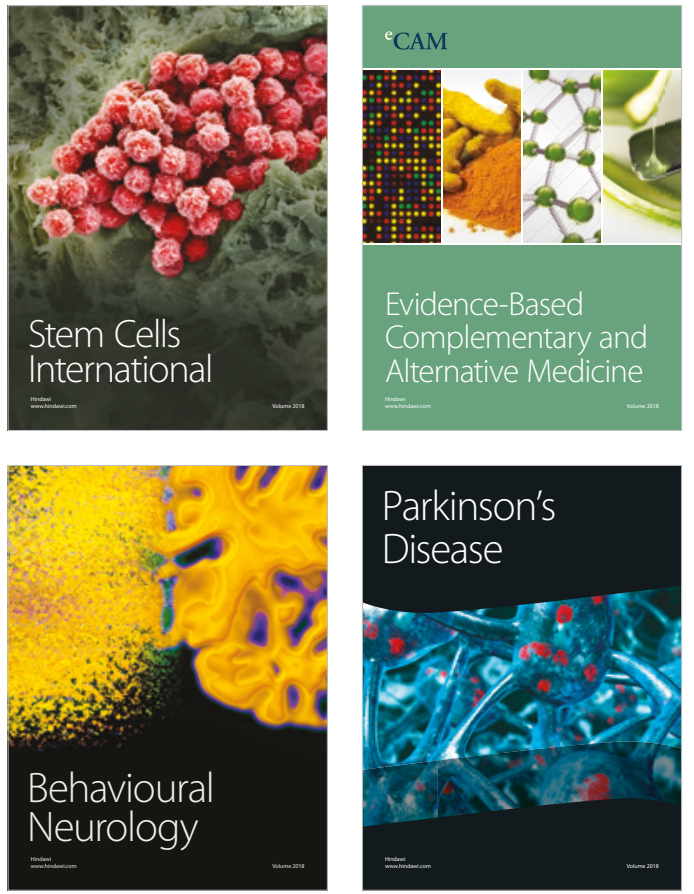

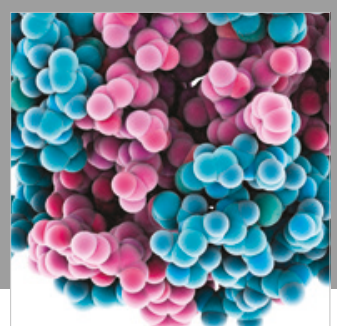

ournal of

Diabetes Research

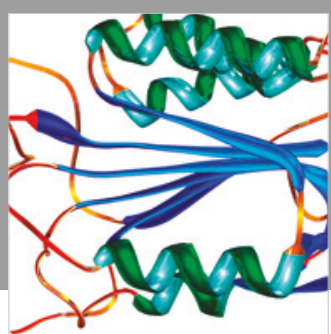

Disease Markers
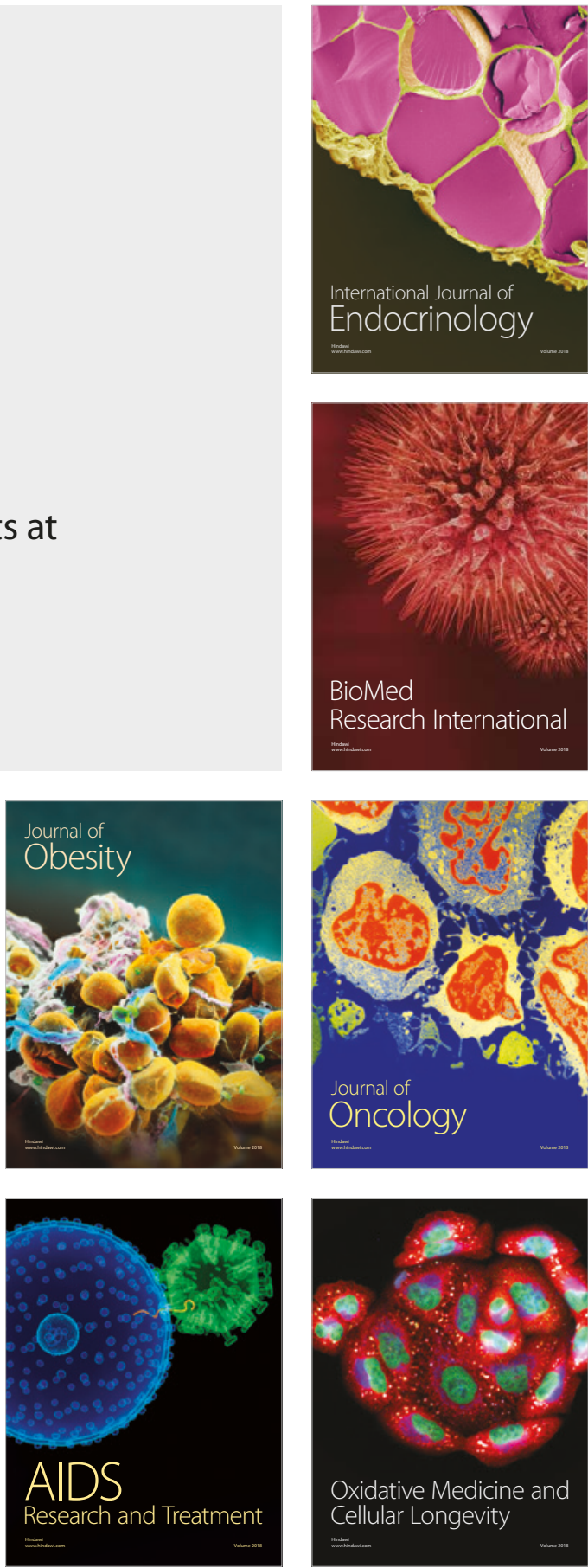